DAS PROGRAMM

\title{
VERFASSUNG UND RECHT IN ÜBERSEE
}

Von Prof. Dr. Herbert Krüger

\section{A. Bestandsaufnahme}

Eine Zeitschrift, die sich mit „Verfassung und Recht in Übersee“ beschäftigen will, bedarf keiner Rechtfertigung. Wenn die sogenannte „Völkerfamilie“ im letzten Jahrzehnt Dutzende von neuen Staaten in ihre Mitte aufgenommen hat und wenn diese Völker schon nach kurzer Zeit eine bedeutsame Rolle in der Weltpolitik spielen, dann ist es eine selbstverständliche Schuldigkeit der Staats- und Rechtswissenschaften, ihre Aufmerksamkeit auf diese Staaten auszudehnen, wobei der Akzent auf „Staaten“ liegt: Denn nach der Gewinnung der politischen Selbständigkeit kann es sich nicht mehr nur darum handeln, diese Völker zu studieren, um etwa eine Typologie primitiver Gesellschaftsstrukturen $\mathrm{zu}$ erarbeiten oder folkloristische Seltsamkeiten zu erfassen.

Mit der Akzentuierung der Staatlichkeit ist zugleich der wesentliche Zug angesprochen, der das Ende der Weltherrschaft Europas von dem Ende der Herrschaft Roms über den orbis terrarum unterscheidet. Abgesehen von Spanien, Portugal und vor allem Rußland' hat die Herrschaft der Europäer über ihre Kolonien oft nur einige Jahrzehnte gewährt. Selbst wenn eine Assimilation im großen Stile beabsichtigt gewesen wäre ${ }^{2}$, wäre eine solche Zeitspanne zu klein gewesen, um so vollständig und endgültig wirken zu können, daß nach der Erlangung der Freiheit die Rückkehr zu den vorkolonialen Zuständen und Auffassungen an Vorhandenes nicht mehr hätte anknüpfen können. Entscheidend sowohl für die Wirklichkeit wie das Programm einer staats- und rechtswissenschaftlichen Beschäftigung mit den neuen Mitgliedern der Völkergemeinschaft ist daher die Tatsache, daß sie die Erlangung der Freiheit gerade nicht als die Chance betrachteten, vorkoloniale Zustände fortzusetzen oder sie gar wiederherzustellen. Diese neuen Gemeinwesen sind vielmehr ausnahmslos und entschieden entschlossen, sich in jeder Hinsicht zu "modernisieren“, d.h. also die Eigenart ihrer alten Herren nicht abzuschütteln, sondern sie sich nunmehr aus eigenem Entschluß und durch eigenes Tun erst vollständig und endgültig zu eigen $\mathrm{zu}$ machen. $\mathrm{Man}^{3}$ hat daher treffend davon gesprochen, daß Europa erst von dem Augenblick an zum Vorbild für die nichteuropäische Welt geworden ist, zu dem es die politische Herrschaft über diese Welt hat aufgeben müssen.

Konkretisiert man diese Feststellung unter dem Blickwinkel des Programms dieser Zeitschrift, dann kann man mit Franz Schurmann"sagen: "The industrial revolution

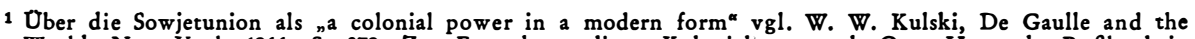
World, New York 1966, S. 379. Zur Entstehung dieses Kolonialismus vgl. Otto Hoetzsch, Rußland in Asien, Stuttgart 1966. Zu alledem ferner R. Pipes, The Formation of the Soviet Union, 2 Auflage, Cambridge, Mass., 1964; und E. Allworth (ed.), Central Asia - A Century of Russian Rule, New York 1967.

2 Wie man dies übereinstimmend als Ziel der französischen Kolonialherrschaft bezeichnet.

s Vl. Rupert Emerson, From Empire to Nation, Cambridge, Mass., 1960, S. VII: "The frontiers of Western Control of the World have been pushed back since 1945; at same time-particularly if Communism be taken as an aberrant creed of Western origin-has scored an extraordinary triumph in that it is more than ever the model to which other countries look in their drive for development." that it is more than ever the model to which other countries look in their drive for development."
Walter Hildebrand, Siegt Asien in Asien?, Göttingen 1966, S. 20: "Die Europäisierung unserer Erde beginnt Walter Hildebrand, Siegt Asien in Asien?, Göttingen 1966, S. 20: "Die Europäisierung unserer Erde beginnt
ja erst in ihrem ganzen Ausmaß, nachdem die äußere Herrschaft der weißen Kolonialherren ihr Ende gefunden hat."

- Ideologie and Organization in Communist China, Berkeley, Calif., 1966, S. XXIX.
} 
in England started as a process of economic transformation that has now reached to the farthest corners of the globe. The French Revolution sowed the seeds of a process of political revolution that has given rise to the modern nation-state. The results of these two revolutions have become the goal of the world's new countries: economic development and political integration." Damit ist gesichert, daß die Aufgabe dieser Zeitschrift sich nicht auf die Vermittlung von Beobachtungen zu beschränken braucht. Die Orientierung an Europa macht es vielmehr möglich, diese Beobachtungen sowohl miteinander wie mit dem europäischen Vorbild zu vergleichen, also insbesondere aus einer solchen Verfassungsvergleichung Nutzen für eine Verfassungslehre zu ziehen. Die Vergleichung der neuen Staaten untereinander sieht sich angesichts dieser Feststellung nicht in der fatalen Lage, völlig Ungleiches und damit im Grunde Unvergleichbares miteinander vergleichen zu wollen: Die Gemeinsamkeit der Orientierung am europäischen Vorbild schafft auf jeden Fall diejenige Ähnlichkeit, die eine Vergleichung nicht als ein von vornherein sinnloses Beginnen erscheinen läßt. Vor allem aber wird hierdurch eine Vergleichung der neuen Gestaltungen mit der europäischen Vorlage erst fruchtbar: Jetzt nämlich erweist es sich als möglich, der praktisch und theoretisch gleich gewichtigen Frage nachzugehen, ob die Orientierung an europäischen Modellen wirklich zu einer Europäisierung nicht nur der Bezeichung nach, sondern in der Sache geführt hat und führen wird.

Nicht abwegig erscheint es, auch der Geschichte der politischen Ideen einige Aufmerksamkeit zu widmen, da es nicht auszuschließen ist, daß sich hierdurch der Blick für die Eigenart der Ergebnisse der Modernisierung um so eher schärfen könnte als man betont, daß Modernisierung nicht Bruch mit der Tradition bedeuten solls.

Es gibt bereits eine ganze Reihe von Werken, die in den soeben umrissenen Zusammenhang gehören. Es überwiegen bei weitem diejenigen Arbeiten, die sich entweder mit einem Land oder innerhalb eines Landes mit einem speziellen Thema beschäftigen. Als Beispiel für die erste Art dieser Gattung sei etwa genannt Crawford Youngs "Politics in the Congo - Decolonization and Independence "6, als Beispiel der zweiten Art A. H. Hanson „The Process of Planning - A Study of India's Five Year Plans 1950-1964"7. In diesem Zusammenhang verdienen Berücksichtigung auch die Verfassungsgeschichten einzelner Staaten wie etwa Claire Palleys „Constitutional History and Law of Southern Rhodesia 1888-1965“8. Diese Zeitschrift sieht nicht ihre letzte Aufgabe darin, mit derartigen Werken bekannt zu machen. Denn man hat den Eindruck, daß alle diese Bemühungen noch zu sehr nebeneinander operieren: So zulässig und notwendig die Beschäftigung mit einem bestimmten Land oder einem speziellen Thema innerhalb eines Landes ist, so könnte doch der wissenschaftliche Gewinn solcher Unternehmen gewiß ohne großen Aufwand nicht unbeträchtlich gesteigert werden, wenn die Autoren gelegentlich einen „Blick über den Zaun" werfen würden. Einen solchen Wunsch kann man aber an die Autoren nur dann richten, wenn man ihnen seine Erfüllung angemessen erleichtert. Solche Erleichterungen möchte diese Zeitschrift zur Verfügung stellen, um dadurch einen Beitrag zur Behebung des Mangels an Querverbindungen zu leisten.

\footnotetext{
5 Vgl. hierzu vor allem T. O. Elias, Government and Politics in Africa, 2. Aufl., London 1963 Als Beispiel für eine Ideengeschidite sei genannt B. A. Saletores „Ancient Indian Political Thought and Institutions“, London 1963.

- Princeton 1965.

7 London 1966.

8 Oxford 1966.
} 
Gemessen an der Gattung der Spezialarbeiten in den erwähnten zwei Verzweigungen befindet sich die Vergleichung in einem sehr erheblichen Rückstand. Gabriel A. Almond und James S. Coleman haben ihr Werk "The Politics of the Developing Areas" 9 bezeichnet als "the first effort to compare the political systems of the developing areas and to compare them systematically according to a common set of categories". Zur etwa gleichen Zeit allerdings hat Edward Shils eine recht ergiebige Skizze „Political Development in the New States"10 vorgelegt, die mir vor allem die Gefahr zu vermeiden scheint, die Erarbeitung von wirklichen Gemeinsamkeiten durch Deduktion zu ersetzen. Kaum der Erwähnung bedarf es, daß in beiden Arbeiten die iberoamerikanischen Staaten nicht berücksichtigt sind.

Man kann sich sehr wohl fragen, ob nicht jedenfalls einstweilen umfassenden Unternehmungen der eben vorgeführten Art solche Arbeiten vorzuziehen sind, die die Vergleichung entweder auf wenige Staaten oder auf ein bestimmtes Thema oder auf beides zugleich beschränken. Die erste Art wird gut belegt durch Eugen Fehrs „Demokratische Staatsformen in Westafrika"11. Es überwiegt jedoch die Kombination beider Beschränkungen. Unter diesem Vorzeichen stehen etwa Ruth SchachterMorgenthaus "Political Parties in French-Speaking West Africa"12 und R. L. Watts" "New Federations - Experiments in the Commonwealth"13; vor allem aber auch die zahlreichen Symposien, auf die weiter unten hingewiesen werden wird.

Besonders erwünscht, ja geradezu unentbehrlich sind natürlich diejenigen Arbeiten, die von Angehörigen der neuen Staaten selbst abgefaßt worden sind. Denn vielleicht sieht der fremde Beobachter manches unbefangener und unbeteiligter; aber er vermag das nicht zu sehen, was man nur "von innen“ wahrnehmen kann. Zeugnisse des Selbstverständnisses sind daher unerläßlich für jede tiefere Deutung des Gegenstandes: Gegenstand der Vergleichung sind ja nicht objektive Gegebenheiten, sondern die Menschen, ihr Geist und ihre Seele, insofern sie mit dieser ihrer Ausstattung diese immateriellen Gegebenheiten über die Texte hinaus zur Existenz bringen und ihnen Inhalt und Sinn verleihen. Leider sind solche Selbstzeugnisse noch sehr selten. Als vorbildlich mag hier wenigstens eines dieser Werke genannt werden: Es ist K. M. Pannikars „The Foundations of New India"14.

Angesichts dieses Ergebnisses unserer Bestandsaufnahme läßt sich nicht behaupten, daß die Beschreibung und Vergleichung von Verfassung und Recht in Übersee der Bedeutung dieses Gegenstandes genüge. Offensichtlich ist das Gegenteil der Fall. $\mathrm{Zu}$ dieser Aktualität des wissenschaftlichen Anliegens tritt hinzu ein noch aktuelleres praktisches Bedürfnis: Man kann sich schwer vorstellen, daß die alten Staaten sich gegenüber den neuen Staaten sachgerecht verhalten und entscheiden könnten ohne über eine, durch umfassende Vergleichung vertiefte Kenntnis von ihnen zu verfügen.

\footnotetext{
Princeton 1960.

10 Den Haag 1962. Inzwischen 2. Auflage 1966; vgl. ferner Heinrich Herrfahrdt, Staatsgestaltungsfragen in Entwicklungsländern Asiens und Afrikas, Karlsruhe 1965, Hans-Ulrich Scupin (Herausgeber), Unvollendete Demokratien, Köln und Opladen 1965.

11 Zürich 1965.

12 Oxford 1964

3 Oxford 1966. Dieser Autor hat Indien, Pakistan, Malaya und Malaysia, Nigeria, Rhodesien und Nyassaland sowie Westindien als vergleichend zu untersuchende Bundesstaaten gewählt. Ziel der Vergleichung ist die Klärung der Motive dieser Verbindungen, ihrer Voraussetzungen, der zur Bewältigung der Probleme gefundenen Lösungen u. a. m. Die Systematik des Vorgehens erhebt dieses Werk zu de $r$ Darstellung des gefundenen Lösungen u. a. m.

14 London 1963. In "diesem Zusammenhang ist auch Alex Quaison-Sackey, Afrika ohne Fesseln - Gedanken eines afrikanischen Politikers, Hamburg 1965, zu erwähnen.
} 


\section{B. Umschreibung der Themen, die die ständige Aufmerksamkeit der Verfassungs- vergleichung verlangen}

Das Feld, dem die Aufmerksamkeit dieser Zeitschrift gelten soll, ist mit „U b e r -

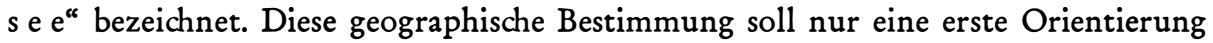
vermitteln. Im entscheidenden soll "Übersee" eine staats- und rechtswissenschaftliche Erheblichkeit von ganz bestimmter Art ansprechen und zusammenfassen. Es handelt sich hierbei nicht eigentlich um das Moment der "Unterentwickeltheit" als weiterer Beitrag zum Thema "Entwicklungshilfe“ wäre diese Zeitschrift überflüssig. Aber auch das Kriterium der "Neuheit“ bezogen auf die Dekolonisation des letzten Jahrzehnts trifft nicht recht das, worauf es entscheidend ankommt. Das spezifische wissenschaftliche Interesse, das die unter dem Merkmal „Úbersee“ ausgewählten politischen Gebilde kennzeichnet und durch diese Gemeinsamkeit als eine besondere Gruppe hervortreten läßt, liegt im Entschluß der in diesen Gebieten zur Führung gelangten Persönlichkeiten und Gruppen zu einer umfassenden und tiefgreifenden Veränderung, die ihrem Telos nach „Modernisierung" und ihrem Modus nach „Revolution" mit dem Ziel einer radikalen Veränderung und Verbesserung der gesellschaftlichen Welt ist. Das bedeutet, daß vor allem in diesen Kreis nicht gehören die Vereinigten Staaten von Nordamerika, daß hingegen in ihn neben Afrika und Asien hineinzunehmen sind die iberoamerikanischen Staaten. Hiermit ist allerdings dieser Kreis so weit gezogen, daß man sich fragen muß, ob er nicht zu weit gezogen ist. Aber so verschieden alle diese Gebilde auch immer sein mögen, es läßt sich nicht verkennen, daß ihnen das Merkmal der Modernisierung durch Revolution gemeinsam ist. Naturgemäß bleiben die Voraussetzungen, unter denen der Entschluß zur Modernisierung gefaßt und ausgeführt wird, himmelweit verschieden, und erst recht läßt sich dies von den Ergebnissen dieses Unternehmens voraussagen: Beides darf die Wissenschaft keinen Augenblick aus den Augen verlieren. Aber alles dies ändert nichts daran, daß dieser Prozeß weithin ein gleicher ist und daher Beobachtung und Würdigung auch unter diesem Blickwinkel erheischt, ganz abgesehen davon, daß erst auf dieser Grundlage wiederum die Verschiedenheiten deutlich und greifbar hervortreten. Will sich die Vergleichung nicht von vornherein um ihre besten Früchte bringen, dann darf sie sich dieser Dialektik von Gleichheit und Verschiedenheit nicht zugunsten der einen oder der anderen Komponente entschlagen.

Hiermit ist das Feld räumlich abgesteckt, dem die Aufmerksamkeit dieser Zeitschrift gewidmet sein soll. Wie der Titel sagt, soll dieses Feld gegenständlich auf „V e r f a s u n g und $\mathrm{R} \mathrm{e} \mathrm{ch} \mathrm{t"} \mathrm{beobachtet} \mathrm{werden.} \mathrm{Unter} \mathrm{Verfassung} \mathrm{kann} \mathrm{nicht} \mathrm{ledig-}$ lich der Verfassungstext verstanden sein. Gerade hier kann, ja müßte die Beschränkung auf Normen und Bezeichnungen ein völlig falsches Bild ergeben. Um dies zu vermeiden, wird man seinen Blick auch und vor allem auf die Verfassungswirklichkeit zu richten haben, damit die wahrhaft wirksamen Faktoren und Kräfte von Staats- und Verfassungsleben erfaßt werden. «Les schémas constitutionels ne redent pas compte de la réalité.»15 So mag es etwa sein, daß ein Gebilde, das sich selbst als Demokratie bezeichnet, sich bei näherer Besichtigung als autoritäre Diktatur erweist ${ }^{16}$.

Neben „Verfassung“ soll „R e ch t" nicht eigentlich einen selbständigen Gegenstand bilden: Diese Zeitschrift ist nicht der Rechtsvergleichung schlechthin gewidmet.

\footnotetext{
15 So Seydou Madani Sy, Recherches sur l'exercice du Pouvoir Politique en Afrique Noire (Côte d'Ivoire, Guinée, Mali), Paris 1965, S. 12.

16 Fehr, a. a. O., S. 5.
} 
Es läßt sich jedoch nicht übersehen, daß die Rechtsordnungen aller Staaten der Welt heute Bestandteile aufweisen, die man der Verfassung zuordnen muß, weil eine Beschränkung auf die Verfassung im formellen Sinne auf jeden Fall ein unvollständiges Bild ergeben müßte. Es handelt sich hierbei um jene Bereiche der Rechtsordnung, die man aus der reinen Privatsphäre auzuklammern und einem Raum des Offentlichen zuzuweisen pflegt, und zwar oft unter der Bezeichnung „Sozialrecht". In Betracht hierfür kommen vor allem Bodenrecht (und dessen Reform!), Wirtschaftsrecht und Arbeitsrecht. Mag hier auch Entscheidendes ungeklärt sein, so ist doch die Tendenz dieses Vorgehens richtig: Eine Verfassungsvergleichung, die sich auf die "politische" Verfassung beschränken und sich nicht Agrar-, Wirtschafts- und Gesellschaftsverfassung einbeziehen würde, wäre einWiderspruch in sich.

Es bedarf keiner Worte, daß es der Wissenschaft sowohl bei der Beschäftigung mit den Normen wie vor allem bei der Aufdeckung der Wirklichkeit nur um Beobachtung und nicht um moralische Wertung gehen kann. Zur Souveränität der Staaten gehört auch die Freiheit der Wahl der politischen, der gesellschaftlichen und nicht zuletzt der ökonomischen Verfassung. Wegen des Ergebnisses einer solchen Wahl steht Dritten weder das Recht der Intervention noch das der Zensur zu. Da derartige Entschließungen nicht allein Sache des guten Willens, sondern weithin von den "Lagen" bestimmt sind, muß es einer wissenschaftlichen Betrachtung vor allem darauf ankommen, die vorgegebenen Ursachen derartiger Entschließungen aufzudecken und sichtbar zu machen. Daß sie gerade in dieser Hinsicht nichts Wesentliches wird feststellen können, wenn sie die Sachverhalte durch die westliche Brille betrachtet ${ }^{17}$, wird anders als die Wertungsfreiheit weithin anerkannt und beachtet.

Nachdem das Arbeitsfeld räumlich und gegenständlich abgesteckt und der freie Blick des Beobachters gesichert ist, ist es möglich, in die Behandlung der Hauptsache einzutreten: Es handelt sich um die Klärung der Frage, welchen Themen der staatsund rechtswissenschaftliche Beobachter seine Aufmerksamkeit vornehmlich zuteil werden lassen soll.

\section{Allgemeine wissenschaftliche Anliegen}

1. Als erste scheint sich die Frage aufzudrängen, wie es eigentlich dazu gekommen ist, daß das soeben umschriebene Feld zur Entstehung gelangte und nun die Beachtung der Wissenschaft fordert. Damit sind die Themen „Kolonialismus“ und „Dekolonisation" angerührt. Da es dieser Zeitschrift jedoch nicht so sehr um die Genesis als vielmehr um die Aktualität gehen soll und dies vor allem für das Dasein der zu beobachtenden Staaten gilt, kommen Kolonisation und selbst Dekolonisation für uns nur insoweit in Betracht, als ihre Folgen sich heute noch bemerkbar machen. Dies ist allerdings an vielen und vor allem an entscheidenden Stellen der Fall. Als Beispiel sei lediglich auf die Tatsache hingewiesen, daß die Grenzen der neuen Staaten genau dieselben sind wie die der alten Kolonialherrschaften ${ }^{18}$.

2. Zum Unterschied von dem allgemeinen geschichtlichen Werdegang muß es der Verfassungs- und Rechtsvergleichung vor allem auf die Aufdeckung geistiger Zusammenhänge ankommen - also um jenen Austausch von Ideen, zu dem sich die

17 Vgl. hierzu etwa E. Stahn, Kommunistische Modelle für Afrika?, Hannover 1967, S. 10; das entsprechende gilt natürlich auch für den "Osten ${ }^{*}, 2$. B. gesellschaftliche Spannungen kurzerhand als "Klassenkampf* zu deuten. Vgl. hierzu Hanson, a. a. O., S. 243.

18 Vgl. zu den hierdurch aufrechterhaltenen ethnischen Spaltungen Claude E. Welch jr., Dream of Unity, Ithaca, N. Y., 1966. 
neuen Völker dadurch bekannt haben, daß sie sich nicht zu Restauration, sondern $\mathrm{zu}$ Modernisierung entschlossen. In diesem Rahmen tritt als erstes und wichtigstes hervor das Phänomen der $\mathrm{R} \mathrm{e} \mathrm{z} \mathrm{e} \mathrm{p} \mathrm{t} \mathrm{i} \mathrm{o} \mathrm{n}^{\mathbf{1 9}}$ - die Übernahme nicht nur einzelner fremder Gedanken, sondern eines vollständigen Ideensystems, ja vielleicht sogar einer ganzen Denkweise. Geschichtsphilosophen und Rechtstheoretiker sehen sich heute durch die unvergleichliche Chance begünstigt, das Phänomen der Rezeption nicht an einem geschichtlichen Vorgang (etwa der Rezeption des römischen Rechts durch die Deutschen) studieren zu müssen - vielmehr eine Fülle solcher Rezeptionen „in actu“ als lebendiges Ereignis beobachten und auswerten zu können. Diese Möglichkeit erhält zudem dadurch eine eigenartige Note, daß die Übernehmer sich dem Angebot zweier "Welten" gegenübersehen, die miteinander um die Übernahme wetteifern: Es ist dies der „Friedliche Wettbewerb“ um die „Dritte Welt“, der nach russischer Deutung das Wesen der „Friedlichen Koexistenz" ausmacht ${ }^{20}$. Wenn es sich hierbei auch um ein Moment der Politik handelt, so beeinflußt es doch die aktuellen Rezeptionen so stark, daß auch eine wissenschaftliche Betrachtung es nicht ganz übergehen kann ${ }^{21} 22$.

Einige in diesem Zusammenhang sich stellende Probleme sollen wenigstens angedeutet werden. Zum ersten kann es zweifelhaft sein, ob eine Rezeption überhaupt möglich ist. Rezipiert werden kann ja niemals eine vollständige Wirklichkeit hierfür kommen ausschließlich Ideologien, Modelle, Institute und Gestaltungen in Betracht. Sie müssen von einer Wirklichkeit abgelöst werden, zu der sie in einem dialektischen Verhältnis wesentlicher Zusammengehörigkeit stehen und einer andersartigen Wirklichkeit in einem entsprechenden Verhältnis zugeordnet werden. Diese Wirklichkeit, insbesondere z. B. die Mentalität des aufnehmenden Volkes mag sich so sehr gegen das rezipierte Geistesgut durchsetzen, daß dieses seine Eigenart verliert und allenfalls eine Fassade zu bilden mag, hinter der sich eine inadäquate Realität verbirgt. Eine derartige Verwandlung verurteilt die Rezeption zur Sinnlosigkeit, insofern es ihr gerade darum gehen muß, zwar fremdes $\mathrm{zu}$ inkorporieren, aber eben als fremdes, das seinerseits die eigene Wirklichkeit bestimmen soll.

Man kann, wie es Sy ${ }^{23}$ tut, die Widerstandskraft und die Umgestaltungsfähigkeit der eigenen Wirklichkeit so hoch einschätzen, daß eine echte und sinnvolle Rezeption nicht mehr möglich erscheint. Es ist anzunehmen, daß dieses Urteil zu negativ ist. Es läßt sich nicht verkennen, daß eine jede Rezeption ein anderes Element in ein bisheriges Leben überträgt („transplantiert“), das nach einem solchen Vorgang doch nicht mehr dasselbe ist, das es vorher war. Jedenfalls kann man eine solche Möglichkeit nicht von vornherein ausschließen. Insbesondere wird man die Völker nach Rezeptionsfähigkeit und Rezeptionswilligkeit zu differenzieren haben. Man muß aber auch damit rechnen, daß sich Völker über die Widerstandskraft ihrer Eigenart irren: Entschließen sie sich z. B. zur Übernahme der „Industriellen Revolution“, dann kann es gar nicht ausbleiben, daß sich nicht nur ihre äußere Lebensweise, sondern auch und vor allem ihre Geistesverfassung ändert ${ }^{24}$.

Ist geklärt, als was der aufnehmende Staat fremdes Geistesgut rezipiert hat, dann stellt sich als zweite die Frage, was er rezipiert hat. Hat er z. B. die Einrichtungen

19 Hierzu grundlegend Franz Wieacker, Privatrechtsgeschichte der Neuzeit, 2. Aufl., Göttingen 1967, S. 125 ff.

20 Vgl. hierzu etwa Frederick C. Barghoorn, Soviet Foreign Propaganda, Princeton 1964, S. $98 \mathrm{f}$.

21 In den letzten Jahren ist China als Anbieter von Ideen in den Kreis der Konkurrenten eingetreten, die sich um Ubernahme ihres Geistesgutes durch die sich modernisierenden Staaten bemühen.

22 Vgl. hierzu das früher bereits erwähnte Werk von E. Stahn.

${ }^{23}$ A. a. O., S. 12: "L'étude révélera l'inadaptation des modèles occidentaux aux réalités africaines."

24 Zur Ubertragbarkeit von Kultursystemen vgl. auch Talcott Parsons, The Social System, London 1951 Nadıdruck 1964, S. 15. 
und Rechtsregeln der englischen Verfassung übernommen, dann kann zweifelhaft sein, ob er hiermit auch deren „ambiance“, insbesondere deren Konventionen übernommen hat ${ }^{25}$. Eine ähnliche Frage ist hinsichtlich der Verfassungsauslegung aufzuwerfen. Hier können sich vornehmlich zwei Zweifel einstellen: Sind mit einer Verfassung auch die Ergebnisse von deren Auslegung, insbesondere also auch die Entscheidungen der Verfassungsgerichte übernommen und gleichgültig, wie immer man diese erste Frage beantworten mag: Sind mit einer Verfassung auch die spezifischen Methoden von deren Auslegung rezipiert ${ }^{26}$ 27?

Verfassungsvergleichung dient nicht zuletzt den Zwecken der Selbstkritik, und zwar gerade auch der Selbstkritik der Anbieter von zu rezipierendem Geistesgut. Zahl und Maß der Rezeptionen müssen von ihnen als Beurteilung der Brauchbarkeit und vor allem der Modernität ihrer Einrichtungen und Ideologien gewertet werden. Eine entsprechende Statistik ${ }^{28}$ w ürde somit nicht etwa nur die Neugierde oder die Eitelkeit befriedigen. Es bedarf nicht einmal einer solchen Statistik für die Feststellung, daß Deutschland als Exporteur von Geistesgut an letzter Stelle liegt, wenn es nicht sogar hierfür gänzlich ausfällt. Vom Grundgesetz interessieren allenfalls das sogenannte „konstruktive Mißtrauensvotum“29 und die Formulierung des Grundrechtskatalogs $s^{30}$, aus der Gesellschaftsverfassung die „dynamische Rente“. Mag dies darauf zurückführen, daß Deutschland im Augenblick der Befreiung seit Jahrzehnten Kolonialmacht nicht mehr gewesen ist. Des Nachdenkens über die Ursache dieser negativen Bilanz sind die Deutschen jedoch keineswegs überhoben.

3. Letztes Ziel sowohl aller gegenwärtigen Rezeptionen wie ihr entscheidendes

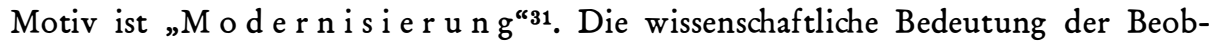
achtung und Deutung dieses Gegenstandes an sich bedarf keiner Hervorhebung. Diese Feststellung wird von zwei Überlegungen erhärtet. Sie betrifft einmal diejenigen Staaten, die bereits modern sind. Hier gilt ganz allgemein, was früher speziell für die Rezeption gesagt worden ist: Diese modernen Staaten haben die Möglichkeit, durch die Beobachtung der Modernisierung der neuen Staaten Licht auf den Hergang ihrer eigenen Modernisierung zu werfen, ohne daß damit diese Prozesse als identisch bezeichnet werden sollen. Die neuen Staaten hingegen können aus der Geschichte der Modernisierung des Westens den Trost schöpfen, daß auch hier die Modernisierung unter den größten Schwierigkeiten erkämpft werden mußte ${ }^{32}-$ bis hin zu der Arbeitsdisziplin, die eine industrialisierte Wirtschaft voraussetzt ${ }^{33}$. Dieser Rückblick mag vielleicht davor warnen, in einigen wenigen "großen Sprüngen" das Ziel der Modernisierung erreichen zu wollen oder vor "compressing the work of centuries almost into decades"34.

25 Vgl. hierzu Young, a. a. O., S. 326; Palley, a. a. O., S. 413; Fehr, a. a. O., S. 123.

26 Vgl. hierzu Klaus Friedrich Arndt, Parlamentarische Geschäftsordnungsautonomie und autonomes Parlamentsrecht, Berlin 1966, S. 46.

$27 \mathrm{Zu}$ alledem ist heranzuziehen G. Burdeau, Traité de Science Politique II, L'Etat, 2. Aufl., Paris 1967, S. 17, Ziffer 9. Dieser Autor weist zutreffend auf die Möglichkeit hin, daß rezipierte Gehalte und Gestaltungen wie insbesondere der Moderne Staat in ihrer neuen Umgebung die gleichen Kämpfe um ihre Durchsetzung gegen eine "feudale ${ }^{*}$ Welt auszufechten haben werden, wie sie dies in ihren Entstehungsländern haben tun müssen.

28 Unter diesem Blickwinkel müssen auch die großen Persönlichkeiten der Befreiung gewürdigt werden. Nehru z. B. hat sich nach Michael Brecher, Nehru - A Political Biography, London 1959, nur für drei Ereignisse interessiert: Die amerikanische, die französische und die russische Revolution.

$29 \mathrm{Vgl}$. Eugen Fehr, a. a. O., S. 71.

so Vgl. Enno Kliesch, Der Einfluß französischen Verfassungsdenkens a uf afrikanische Verfassungen, Hamburg 1967 (Beiheft 1 zu dieser Zeitschrift), S. 27.

31 Zum Unterschied von modernization " und „westernization ${ }^{*}$ vgl. Donald M. Lowe, The Function of "China“, in: Marx, Lenin and Mao, Berkeley, Calif., 1966, S. XIII, Anm.: “... The word "modernization" implies a model of technical efficiency, whereas the word 'westernization' implies a model of Western rational values..." Der zweite Terminus und seine Definition dürfen allerdings nicht zu dem Irrtum verleiten, die östliche Ideologie sei nicht auch eine wwestliche“; vgl. hierzu oben R. Emerson.

32 Vgl. zur Modernisierung der USA S. M. Lipset, The First New Nation - The United States in historical and comparative perspective, New York 1963.

ss Vgl. hierzu R. Goetz-Girey, Croissance et Progrès a l’Origine des Sociétés Industrielles, Paris 1966, S. 37.

s4 T. O. Elias, a. a. O., S. 54. - Dies alles gilt natürlich erst recht, wenn die natürlichen Umstände „are 
„Modernisierung“ ist zweitens vor allem deswegen geradezu ein Schlüsselthema, weil es zu einer Erkenntnis derjenigen wesentlichen Punkte verhilft, an denen eine Vergleichung anzusetzen hat, wenn sie fruchtbaren Ertrag erzielen will. Erinnert man sich daran, daß es jedenfalls in Kontinentaleuropa der Moderne Staat gewesen ist, der die Modernisierung Europas eingeleitet, betrieben und durchgesetzt hat, dann wird eine gewisse Wahrscheinlichkeit dafür sprechen, daß die dekolonisierten Völker sich für denselben - institutionalisierten - Vorkämpfer ihrer Modernisierung und dementsprechend für die - weil vom Staat bewirkte - friedliche "Revolution von oben" entschieden haben werden. Geht man hiervon jedenfalls als Arbeitshypothese aus, dann wird sich sogleich die Frage stellen, wie es mit den Voraussetzungen für eine solche moderne Staatlichkeit steht und, da diese Voraussetzungen im Zweifel bis hin zur Mentalität nicht vorgegeben sein werden, ob man diesen Mangel erkannt hat und ob und wie man ihm abzuhelfen sucht ${ }^{35}$.

Angesichts der Bedeutung von "Modernisierung" als Leitbild gerade auch für Erfassung und Vergleichung von Verfassungen und Recht der neuen Staaten nimmt es nicht wunder, daß dieses Thema zwei umfassende und gründliche Bearbeitungen gefunden hat ${ }^{36}$. Beide Werke wären an sich eines umfassenden Referates würdig. Dieses Programm muß sich mit einigen Bemerkungen begnügen. Als erstes sei hieraus entnommen, daß die Entscheidung für Modernisierung bereits ein Mindestmaß von Modernität voraussetzt - nämlich die Überzeugung, daß es Alternativen gibt, zwischen denen der Mensch frei wählen könne ${ }^{37}$. Diese Entscheidung ist daher nicht das Ergebnis wissenschaftlicher Erkenntnis, sondern Bekenntnis zu einer "moral ideology"38, die den Weg zur Menschlichkeit über Rationalisierung der Geistesverfassung führen sieht.

Hiermit ist klargestellt, daß sich Modernisierung nicht in einer noch so umfassenden und radikalen Veränderung der äußeren Verhältnisse erschöpft - daß sie vielmehr von Ursprung her eine innere Wandlung des Menschen zum modernen Menschen ebenso voraussetzt wie anstrebt. Modern aber ist der Mensch heute vor allem insofern, als es ihm entscheidend um die Hebung des Lebensstandards durch rationales Wirtschaften geht $^{39}$. Es ist daher der homo oeconomicus ${ }^{40}$, der im Zeichen der Modernisierung die Herrschaft auch in den neuen Staaten antreten und durch diese seine Herrschaft die Hebung des Lebensstandards herbeiführen soll. Naturgemäß findet ein solcher Entschluß zur Modernisierung den "homo oeconomicus“ ebensowenig vor, wie ihn in Europa Merkantilisten und Kapitalisten vorgefunden haben: $\mathrm{Er}$ ist ein Kunstprodukt, das in den neuen Kontinenten ebenso wird gezüchtet werden müssen, wie man ihn seinerzeit in Europa gezüchtet hat und unablässig weiter züchtet. Zwar wird es nur allzugern übersehen, versteht sich aber im Grunde von selbst, daß sich ein solcher Züchtungserfolg nur durch schwere

not favorable to rapid improvement ${ }^{\alpha}$; so das Urteil von Ursula K. Hidks, Development, Finance-Planning and Control, Oxford 1965, S. 7.

ss Die Einsicht in solche Zusammenhänge läßt vielleicht sogar den Kolonialismus in einem etwas anderen Lichte sehen, vgl. hierzu R. Emerson, a. a. O., S. 6: “... A plausible case can, however, be made tor the proposition that the future will look back upon the overseas imperialism of recent centuries, less in terms of its sins of oppression, exploitation, and discrimination, than as the instrument by which the spiritual, scientific and material revolution which began in Western Europe with the Renaissance wis spread to the rest of the world ...

s6 David E. Apter, The Politics of Modernization, Chicago 1965 - ein Buch, das unschätzbare Beiträge zugleich zu einer Verfassungslehre liefert.

Marion J. Levy jr., Modernization and the Structure of Societies - A Setting for International Affairs, Princeton 1966. Hierzu auch die Besprechung durch J. Frankel in: International Affairs 43 (1967), S. 565 $\mathrm{f}$

$37 \mathrm{Da}$ dies keineswegs selbstverständlich ist, daß vielmehr die Vorstellung, daß Alternativen vorhanden und daß eine freie Wahl zwischen ihnen möglich sei, eine moderne, also geschichtsbedingte Vorstellung ist, zeigt Apter, a. a. O., S. $9 \mathrm{ff}$.

38 Apter, a. a. O., S. 319.

so Vgl. zur Planung in den neu entstandenen Staaten z. B. das oben bei Anm. 7 erwähnte Werk.

$4 \mathrm{Vgl}$. hierzu E. L. Godkin, The Economic Man, in: Problems of Modern Democracy, Essays ed. by M Keller, Cambridge, Mass., 1966, S. $156 \mathrm{ff}$. 
Verluste an anderen Stellen erkaufen läßt, so daß vor dem Entschluß auf jeden Fall eine Gewinn- und Verlustrechnung aufgemacht werden sollte. Auf welche Schwierigkeiten eine solche von oben betriebene Ökonomisierung stößt und mit welchen nicht zuletzt moralischen Verlusten ihr Erfolg rechnen muß, zeigt eine Studie von Aníbal Pinto ${ }^{41}$ : Hier wird eine Haltung beschrieben und festgehalten, die eine Verbesserung der Zustände nicht in Betracht zieht, die vielmehr auf dem unveränderten Boden der gegebenen Verhältnisse stehend ausschließlich eine Verbesserung ihres eigenen Loses ins Auge faßt - die also den Gedanken einer Revolution, vor allem auch sozialen Revolution ebensowenig zu denken vermag, wie man ihn in Europa vor der Neuzeit zu denken vermocht hat.

Lediglich erwähnt sei, daß es nicht an Autoren fehlt, die sich dem Thema „Modernisierung" in Gestalt der Behandlung eines konkreten Falles gewidmet haben ${ }^{42}$.

\section{Probleme moderner Staatlichkeit}

Die Staatlichkeit der neuen Staaten fordert doppelte Aufmerksamkeit, weil der Staat zugleich ein Element der Modernität und der Demiurg der Modernisierung ist. Das bedeutet, daß die politische Entwicklung in den neuen Staaten vor allem darauf zu beobachten ist, ob und welche Züge der Verstaatung sie aufweist und insbesondere welche Voraussetzungen moderner Staatlichkeit man zu schaffen sucht.

\section{Weltlichkeit}

Modernisierung bedeutet zuerst Hinwendung zur irdischen Welt und deren Bejahung - sie bedeutet also Verweltlichung oder Säkularisierung. Hier zeigt sich der Staat in seiner Doppelrolle als Element und Faktor der Modernisierung, indem er ebenso wesentlich weltliches Phänomen wie Förderer der Verweltlichung ist. Das letzte äußert sich in der Sache als Ausschaltung aller religiösen Gesichtspunkte aus weltlichen Angelegenheiten, insbesondere aus der Politik. Organisatorisch wirkt sich die Weltlichkeit des Staates in dem aus, was man speziell „Trennung von Kirche und Staat" nennt, was aber ohne weiteres auch gilt für alle anderen Arten von Verflechtung $\mathrm{zwischen}$ religiösen und irdischen Institutionen.

Unter diesen Vorzeichen hat der Versuch einer Übersicht über den Stand der Dinge $1962^{43}$ folgendes ergeben, und zwar zunächst für den Islam: Die grundsätzliche Frage scheint allenthalben entschieden $z u$ sein. Zwar ist allgemein anerkannt, daß der Islam eine Lehre nicht nur für das Verhältnis des Menschen zu Gott ist, sondern daß er Maßgeblichkeit auch für die Regelung der Beziehungen der Menschen untereinander erheischt. Aber hieraus wird durchweg nicht der Schluß gezogen, daß Verfassung, Gesetze und alle anderen Verhaltensnormen an den religiösen Normen

\footnotetext{
1 Political Aspects of Economic Development in Latin Africa, in: Claudio Veliz (ed.), Obstacles to Change in Latin America, London 1965, S. $9 \mathrm{ff}$.

12 Peter Doll, Der senegalesische Weg zum afrikanischen Sozialismus - Konzeption und Verwirklichung der senegalesischen Entwicklungspolitik, Hamburg 1966.

Pierre Fougerolles, Modernisation des hommes - L'exemple du Sénégal, Paris 1967; J. H. Thompson u. a., Modernizaion of the Arab World, Princeton 1966; vgl. auch die Literatur zur Modernisierung Japans und der Türkei, z. B. R. E. Ward und D. A. Rostow (ed.), Political Modernization in Japan und Turkey, Princeton 1964

43 Vgl. Herbert Krüger, Grundzüge der Verfassungsbildung in den neu entstehenden Staaten, in: Deutsche Landesreferate zum VI. Internationalen Kongreß für Rechtsvergleichung in Hamburg 1962, Berlin und Tübingen 1962, S. 309 ff., S. $318 \mathrm{ff}$.
} 
$\mathrm{zu}$ orientieren seien ${ }^{44}$. Eine Ausnahme machen allenfalls Afghanistan ${ }^{45}$, Brunei ${ }^{46}$ und vor allem Pakistan nach der inzwischen außer Kraft gesetzten Verfassung von 1956. In deren Präambel heißt es, daß nach dem Willen des Gründers von Pakistan, Jinnah, Pakistan ein demokratischer Staat auf der Grundlage der islamischen Prinzipien sozialer Gerechtigkeit sein solle, und daß daher hier die Muslims befugt seien, ihr Leben nach den Lehren und Geboten des Islam zu ordnen. Man ${ }^{47}$ hat darauf hingewiesen, daß eine derartige Bindung ein modernes Kreditsystem ausschließe, da der Islam wie das Christentum ein Zinsverbot kenne. Gerade diese Parallele zeigt aber, daß eine solche Festlegung nicht auf ein unübersteigbares Hindernis hinauszulaufen braucht.

Zugleich Bindung an und Herrschaft über die Religion bedeutet es, wenn der weltliche Herrscher zugleich zum Oberhaupt der Gemeinschaft erhoben wird, die die Staatsreligion bekennt. Etwas Derartiges ${ }^{48}$ ist nur noch der Fall in Brunei und in den Einzelstaaten von Malaya.

Eine zweite Gruppe bilden diejenigen Staaten, die zwar eine Staatsreligion erklären, nichtsdestoweniger aber allen anderen Bekenntnissen volle Freiheit gewähren und insbesondere die Staatsreligion nicht zur Richtschnur für die weltliche Gesetzgebung erheben. Den Islam zur Staatsreligion erklären von geltenden Verfassungen Jordan (Transjordanien) ${ }^{49}$, Irak ${ }^{50}$, Malaya ${ }^{51}$ und Mauretanien ${ }^{52}$. Eine Einschränkung der Freiheit anderer Religionen folgert hieraus allein Malaya, indem es die Einzelstaaten der Förderation durch Artikel 11 (4) seiner Verfassung 1957 ermächtigt, die Propaganda anderer Religionen unter den Muslimen zu beaufsichtigen und zu beschränken. Eine gewisse Konkretisierung erfährt das Prinzip der Staatsreligion in denjenigen Staaten, in denen das Personenstandsrecht religiöses Recht ist und demgemäß entsprechende religiöse Gerichtshöfe vorgesehen sind wie in Jordan (Art. 99) und in der alten Verfassung des Irak.

$\mathrm{Da}$ dem Modernen Staat ein Werturteil über die Religionen versagt ist, und er daher eine Religion nicht zur Staatsreligion erheben darf, weil er sie für die richtige hält, sondern dies allenfalls tun darf, weil die überwiegende Mehrheit seiner Bürger die betreffende Religion frei bekennt, verfährt in dieser Hinsicht adäquat die Verfassung von Birma, wenn sie in Artikel 21 (1) bestimmt: "The State recognizes the special position of Buddhism as the faith professed by the great majority of the citizens of the Union." Daß eine dergestalt begründete Staatsreligion der Freiheit anderer Bekenntnisse erst recht keinen Abbruch zu tun braucht, zeigt der folgende Absatz des gleichen Artikels, indem er Christentum, Hinduismus und Animismus als einige der in der Union existierenden Religionen anerkennt.

Indonesien nimmt eine Sonderstellung ein. In der Präambel seiner Verfassung bekennt sich dieser Staat zur Allmacht Gottes und erklärt dann in Artikel 43: "The State is based on the belief in the Divine Omnipotence." Von einem solchen Standpunkt aus kann man naturgemäß nur zu einer jedermann begünstigenden Gewähr-

\footnotetext{
44 Für eine solche Maßgeblichkeit sehr entschieden M. Asad, The Principles of State and Government in Islam, Berkeley and Los Angeles 1961.

45 Verfassung 1931 Art. 1, 5.

46 Verfassung 1959 Art. 3 (1).

47 Vgl. Sir I. Jennings, Problems of the Commonwealth, Durham, N. C., 1958, S. 36.

4 Vgl. Brunei, Verfassung 1959 Art. 3 (2): "The Head of the Religion of the State shall be the Sultan."

Malaya Verfassung 195? Art. 3 (2): "In every State other than Malacca and Penang the position of the

Ruler as the head of the Muslim Religion of the State ... are unaffected and unimpaired..."

49 Verfassung 1952 Art. 2: "Islam shall be the religion of the State."

50 Provisorische Verfassung 1958 Art. 4: "Der Islam ist die amtliche Religion des Staates."

51 Verfassung 1957 Art. 3 (1): "Islam is the religion of the Federation..."

52 Verfassung 1959 Art. 2: «La religion du peuple mauritanien est la religion musulmane.»
} 
leistung der Freiheit der Religion und des Gottesdienstes sowie zu gleichem Schutz für alle Religionsgemeinschaften gelangen, wie dies in den folgenden Absätzen dieses Artikels angeordnet ist.

Unter den Staaten, die ohne Staatsreligion Religionsfreiheit gewähren, hebt sich eine Gruppe heraus, die hierüber hinausgehend sich ausdrücklich für laizistisch erklärt. Es sind dies, mit Ausnahme von Madagaskar, diejenigen Staaten, die aus früheren französischen Besitzungen entstanden sind, und zwar unabhängig davon, ob sie in der Communauté verblieben sind oder nicht ${ }^{53}$. Hierzu ist weiter nichts zu bemerken, als daß gerade die Festlegung auf ein so spezifisch französisches Phänomen wie den Laizismus zeigt, wie sehr man den Unterschied zwischen Wortlaut und Wirklichkeit bei solchen rezipierten Verfassungen im Auge behalten muß. Eine Variante bietet allenfalls Zentralafrika, indem es in der Präambel seiner Verfassung 1959 die Religionsgemeinschaften anerkennt als „facteur d'éducation morale“.

Dieser Querschnitt erfordert eine grundlegende Berichtigung nicht: Die Entwicklung hat sich überall in der damals festgestellten Richtung weiterbewegt. Das gilt vor allem für das Verhältnis zum Islam und seinem Anspruch, Regeln auch für die Ordnung der weltlichen Verhältnisse $z u$ enthalten. Von ihm stellt M. Halpern ${ }^{54}$ fest: "Islam ... as a unique pattern for politics, economics and society is no longer practised" - womit zugleich die weltliche Rolle der Ulemas ausgespielt ist. Pakistan macht keine Ausnahme von dieser Regel. Folgt man der Monographie, die Karl von Vorys ${ }^{55}$ über dieses Land vorgelegt hat, dann ist auch hier die Herrschaft des Islam und seiner Repräsentanten, der Ulema, trotz der religiösen Bestimmtheit des pakistanischen Nationalismus in weltlichen Angelegenheiten praktisch zu Ende gegangen, obwohl es nicht an gelegentlichen Ausbrüchen religiösen Fanatismus gefehlt hat.

\section{Staatsgebiet und Staatsvolk}

Die Theorie beschäftigt sich mit dem Staatsgebiet vor allem unter dem Gesichtspunkt, wie man sich das Verhältnis von Staat und Gebiet vorzustellen habe. Dieses Problem interessiert die Verfassungsvergleichung lediglich insoweit, als es sich um die Verdrängung des „dominium“ von Häuptlingen und Scheichen durch das „imperium" der Allgemeinheit handelt. In dieser Hinsicht bietet wohl nur noch die arabische Halbinsel beachtenswertes Material ${ }^{56}$. Was das Staatsgebiet als solches angeht, so verlangt die Theorie als erstes, daß es "territorium clausum", also eine geschlossene, sowohl von Enklaven freie wie nicht durch Exklaven mißbildete abgerundete Fläche sein soll. Solche Einsprengsel erscheinen dann besonders anstößig, wenn sie sich in der Hand früherer Kolonialherren befinden. In solchen Fällen schreckt man, wie Goa zeigt ${ }^{57}$ ebensowenig wie der Moderne Staat in Europa vor der Anwendung von Gewalt zurück, um die Einheitlichkeit und Geschlossenheit des Staatsgebietes herzustellen. In diesem Zusammenhang gehört auch das Phänomen der militärischen Stützpunkte. Eigentlich nur der Vollständigkeit halber mag zum Thema des Staatsgebietes die Lehre von den „Natürlichen Grenzen“ des Staates

\footnotetext{
53 So heißt es auch in der Verfassung 1958 von Guinea Art. 1: «La Guinée est une République Démocratique, Laïque et Sociale.»

Vgl. hierzu Sékou Touré, Expérience Guinéenne et Unité Africaine, Paris o. J. (etwa 1961).

54 The Politics of Social Change in the Middle East and North Africa, Princeton 1963, S. 128.

55 Political Development in Pakistan, Princeton 1965, S. 56, 129 f. Nachzutragen ist hierzu L. Binder, Religion and Politics in Pakistan, Berkeley 1961.

- Vgl. etwa die soeben durch die Errichtung des Staates Südjemen untergegangenen Sultanate.

57 Vgl. L. Lawrence, Nehru seizes Goa, New York 1963.
} 
erwähnt werden: Ihrem Auftreten wird ebenfalls ein gewisses Maß an Aufmerksamkeit zu schenken sein. Eine besondere und schwierige Problematik stellt sich in dem Bundesstaat, deren Lösung allerdings durchweg weniger von territorialen als von personalen, insbesondere ethnischen und linguistischen Gesichtspunkten bestimmt wird. Daß ein durch solche Faktoren determinierter Bundesstaat zu einer Gefahr für die Einheit und Allgemeinheit des Staatsvolkes werden kann, hat man vor allem in Indien und jüngst in Nigeria wohl bemerken können.

Der Kampf gegen den Kolonialismus ist seltsamerweise von einem Nationalismus ohne Nationen geführt worden: Er hat gewissermaßen die Nation vorweggenommen und sieht sich nach dem Sieg genötigt, nun die Nation zu bilden, in deren Namen er die Freiheit gefordert hat. Diese Nationen werden daher ebensowenig Naturprodukte sein wie ihr Staat und ihr Gebiet es sind -, man wird sie ebenfalls als Kunstwerke ansprechen müssen. Diese Feststellung ist nicht so befremdlich wie es auf den ersten Blick den Anschein hat. Denn vor allem auch die europäischen Nationen sind durchweg nicht aus der Hand Gottes oder der Natur ohne Zutun des Menschen hervorgegangen, sie sind vielmehr weithin als menschliche Hervorbringungen anzusehen ${ }^{58}$. Das heißt jedoch nicht, daß man die neuen Staaten als "Schmelztiegel" von der Art anzusehen habe, wie es die Vereinigten Staaten gewesen sind und noch sind. Man muß damit rechnen ${ }^{59}$, daß die neuen Staaten davon absehen, sich zum Nationalstaat im Sinne einer Identität von Staat und ethnischer Gruppe zu entwickeln, und sei es auch nur aus der nüchternen Überlegung, daß über einem solchen Versuch eine Einheit zerbrechen könnte, die einstweilen jedenfalls oft nicht viel mehr als ein Residuum der Kolonialherrschaft ist. Eine solche Enthaltung bedeutet, daß man die Allgemeinheit des Staatsbürgertums, diese erste Voraussetzung jeder Staatlichkeit und vor allem ihrer Einheit, nicht in der Gemeinsamkeit einer Nationalität suchen, vielmehr gerade diesen Faktor als Integrationsmoment auszuschalten und diese Art von Besonderheiten gegen ein Einschmelzen zu sichern hat.

Soweit ersichtlich, gibt es nur ein Beispiel dafür, daß ein Staat auf die Bildung eines (im übrigen auch nicht national homogenen) Staatsvolkes, also auf eine Allgemeinheit der Staatsbürgerschaft im qualitativen Sinne verzichtet hat. Es handelt sich um den Libanon ${ }^{60}$. Hier ist der Staat begriffen und verfaßt als eine Vereinigung von Gruppen, und zwar von religiös bestimmten Gruppen, was im Prinzip zur Folge hat, daß der Proporz allenthalben die entscheidende Rolle spielt.

Abgesehen von dieser Ausnahme scheinen alle neuen Staaten auf die Schaffung einer Allgemeinheit auszugehen, die weder ein Pluralismus von Besonderheiten noch der Monismus einer Besonderheit ist, also zwischen der Alternative von Herrschaft oder Unterdrückung der Besonderheiten hindurchzusteuern sucht, indem sie die staatliche Einheit auf anderen Momenten aufbaut, die allerdings von jedermann müssen geteilt und akzeptiert werden können, wenn sie für die Bildung einer Allgemeinheit in Betracht kommen sollen. Im Lichte dieses billigenswerten Entschlusses hat man nicht zuletzt die Politik dieser Staaten zu sehen. So mag ein Europäer Anstoß am Einparteiensystem nehmen, dem sich so viele der neuen Staaten verschrieben haben. Bevor man hierüber ein Urteil fällt, sollte man bedenken, daß ein Staat, in dem es von Besonderheiten aller Art wimmelt und der auf eine Egalisierung dieser Besonderheiten verzichtet hat, um so mehr darauf bedacht sein muß, daß die zentrifugale Tendenz dieser Besonderheiten durch einheits-

58 Vgl. K. W. Deutsch, Nationalism and Social Communication, 2. Aufl., Cambridge, Mass., und London 1966. 50 Und man möchte es hoffen.

60 Vgl. hierzu die aufschlußreiche Darstellung von Charles Rizk, Le Régime Politique Libanais, Paris 1966. 
stiftende Veranstaltungen zum mindesten neutralisiert wird. Würde zu den vielen und mächtigen Besonderheiten noch eine Mehrheit von Parteien hinzutreten, die als Mehrheit nichts anderes als besondere Parteien, wenn nicht sogar Parteien von Besonderheiten sein würden, dann könnte der Zerfall der staatlichen Einheit schwerlich aufgehalten werden ${ }^{61}$, es würde sich das Ergebnis einstellen, das man durch seine Benennung als „Balkanisierung“ mit Recht zum Schreckbild gestempelt hat und energisch zu vermeiden sucht.

In dieser Sicht erscheint es beinahe als selbstverständlich, daß man allenthalben "Tribalismus“, „Kommunalismus“, „Parochialismus" oder wie immer man die Herrschaft der Besonderheiten benannt haben mag, bekämpft und zurückzudrängen sucht. Soweit Kampfziel nicht die Vernichtung der Besonderheiten wie im Falle der „feudalen“ Herrschaftsverbände oder der Kasten ${ }^{62}$ ist, und zwar ohne Rücksicht darauf, daß solche Gebilde weithin staatliche Aufgaben der sozialen Sicherung wahrnehmen ${ }^{63}$, sucht man sie auf jeden Fall zu „kanalisieren“, wenn nicht sogar durch Kanalisierung für die Allgemeinheit nutzbar zu machen. Die Zauberformel, die sich in solchen Situationen anzubieten pflegt, lautet „F ö d e r a lis m u s“.

Zahlreiche und gerade die bedeutendsten der neuen Staaten sind als Bundesstaaten mit zum Teil sehr komplizierten und differenzierenden Strukturen verfaßt, um den Besonderheiten einen eigenen staatlichen Status zu geben und sie dennoch zu größeren, verteidigungs- und leistungsfähigen Ganzheiten zusammenzufassen. Gerade in dieser Hinsicht bieten die neuen Staaten der Verfassungsvergleichung reichhaltiges und ergiebiges Material bis in einzelne Probleme hinein wie die Zuverlässigkeit der Delegation von Zuständigkeiten oder der „Fondswirtschaft"64 ohne Gesetzgebungszuständigkeit. In der Praxis scheinen Schwierigkeiten mit dieser Lösung noch nicht aufgetreten zu sein. Trotzdem wird man sich jetzt schon über die Grenzen ihrer Wirksamkeit klarwerden müssen.

Diese Grenzen liegen im Prinzip darin, daß der Staat seine Herrschaft territorial umschreibt, während die Besonderheiten der Religion, der Nation, der Kaste und dergleichen personal bestimmt sind. Im Prinzip kann daher der Föderalismus Eigenarten nur dann staatlich verfassen und dennoch $\mathrm{zu}$ einem höheren staatlichen Ganzen zusammenfügen, wenn die in Frage stehenden Besonderheiten sich räumlich radizieren lassen. Diese Identität ist aber so gut wie niemals gegeben ${ }^{65}$. Strenggenommen müßte man sie daher durch Umsiedlungen herbeiführen und dann durch Verbot der Freizügigkeit und des Konnubiums erhalten. Derartige Eingriffe aber stehen außer Diskussion. Bei Lichte besehen mag daher der Föderalismus das Zusammenleben mehrerer Besonderheiten in einem Staate dadurch erleichtern, daß das Staatsgefüge ein lockeres und differenzierenderes ist als das des Einheitsstaates. Aber wo verschiedene Nationalitäten friedlich und freundschaftlich in einem Staate miteinander leben, $\mathrm{da}$ ist es nicht eigentlich die Bundesstaatlichkeit, der dieses Ergebnis letztlich zuzuschreiben ist $^{66}$. Es beruht dieses Bild vielmehr vor allem

\footnotetext{
61 Vgl. hierzu T. O. Elias, a. a. O., S. 102.

62 Vgl. hierzu M. N. Srinivas, Caste in Modern India, London 1962.

6s Vgl. Pannikar, oben Anm. 14; Hanson, a. a. O., S. 245.

o Hierzu P. P. Agarwal, The System of Grants-in-Aid in India, London 1960.

65 Selbst in Kanada mit der Konzentration der französisch sprechenden Bevölkerung in Quebec ist daher das Problem durch den Föderalismus nicht vollständig gelöst; vgl. hierzu Ch. Wagley und M. Harris, Minorities in the New World, New York 1958, S. $169 \mathrm{ff}$; The French Canadians; ferner F. R. Scott, French Canada and Canadian Federalism, in: Evolving Canadian Federalism, Durham, North Carolina 1958, S. $54 \mathrm{ff}$.

os Vgl. hierzu Th. Schieder, Idee und Gestalt des übernationalen Staates, in: Historische Zeitschrift 184, 1957, S. $336 \mathrm{ff} ., \mathrm{S} .342 / 343$.

Ferner derselibe, Die Schweiz als Modell der Nationalitätenpolitik, in: Festgabe für Hans Herzfeld, Berlin 1958, S. 489 ff. Hier heißt es S. 489: „Es träfe nicht den Sachverhalt, wenn man sagen würde, die Schweiz habe das Nationalitätenproblem, d. h. das Problem des Zusammenlebens mehrerer Nationalitäten in einem Staate gelöst, sie hat es vielmehr gar nicht entstehen lassen. ${ }^{\alpha}$
} 
darauf, daß sich die größeren Besonderheiten Mäßigung und Beschränkungen zugunsten der kleineren Besonderheiten auferlegen, so daß es diesen Besonderheiten in diesem Bundesstaat besser geht, als es ihnen in einem eigenen Staat oder in dem Nationalstaat ihrer Nation gehen würde. Hieraus ist sicher soviel zu entnehmen, daß das Problem „Mehrere Nationen in einem Staat" durch die Lösungen „Föderalismus" und „Bundesstaat" nicht erledigt ist ${ }^{67}$.

Die Vergleichung wird aber nicht nur die Modifikationen des Modells oder Idealtyps „Bundesstaat" zu erfassen und auszuwerten haben, die sie unter dem Einfluß der eigenartigen Verhältnisse oder der besonderen Notwendigkeiten ${ }^{68}$ erleiden. Sie wird sich auch der kritischen Frage nicht entziehen können, ob es nicht noch eine bessere Lösung für die Bewältigung der Schwierigkeiten gibt, die mit Hilfe des Bundesstaates bewältigt werden sollen. Dem Bundesstaat wird immer die Antinomie anhaften, daß er personale Eigenschaften mit Hilfe des Territorialitätsprinzips zu konstitutionalisieren sucht. Die Einsicht in diese Antinomie legt die Frage nahe, ob es wirklich ausgeschlossen ist, das Personalitätsprinzip in diesem Zusammenhang nutzbar zu machen, das prima facie als das unmittelbare und das adäquate erscheint. Die Föderalisierung eines Staates gemäß dem Personalitätsprinzip würde an sich der Idee der Allgemeinheit, Gleichheit und Unmittelbarkeit des Staatsbürgertums genauso wenig widersprechen wie eine Föderalisierung gemäß dem Territorialitätsprinzip -, vorausgesetzt, daß die personal konzipierten Einzelstaaten ihren Bürgern Gleichheit, Allgemeinheit und Unmittelbarkeit ihrer Zugehörigkeit nicht vorenthalten würden. Mißlich wäre es, daß solche, in einen (territorial fundierten) Gesamtstaat eingebundenen Personalstaaten eines Territoriums und damit einer greifbaren und handlichen Umschreibung ermangeln würden: Die Bürger dieser Staaten wären auf das ganze Gebiet des Gesamtstaates verteilt. Hiermit könnte man sich wohl abfinden, wenn nicht die Einzelstaaten in Ermangelung eines Territoriums den Gesamtstaat einer Wirkung berauben würden, die trotz aller Radizierung der Einzelheiten auf eine Besonderheit das Territorialprinzip herbeiführt, nämlich einer Ausgleichung der trotz aller Gleichheit in der Besonderheit zahlreich vorhandenen Verschiedenheiten im kleinen Kreise ${ }^{69}$. Dieser Mangel könnte bewirken, daß der personale Föderalismus im Gegensatz zum territorialen Föderalismus die Besonderheiten nicht neutralisiert sondern potenziert. Dieses theoretisch gewonnene Ergebnis scheint durch die praktischen Erfahrungen bestätigt zu werden, die man in Zypern mit "Personalkurien" gemacht hat - um von dem Odium ganz zu schweigen, das die Verteilung der Weißen und Schwarzen auf solche Personalkurien durch die Kolonialherren hinterlassen haben mag. Trotz alledem sollte man diese Möglichkeit nicht aus den Augen verlieren und daher das Beobachtungsfeld auf das Auftreten dieses Phänomens unter Aufmerksamkeit halten.

Gelingt weder die Beschränkung der Allgemeinheit auf verallgemeinerbare Momente noch die Konstitutionalisierung der Besonderheiten in einem territorialen oder personalen Föderalismus - kommt es also doch zu einer Identifizierung des Staates mit einer herrschenden Besonderheit, dann stellt sich das alte verfassungs-

\footnotetext{
67 Vgl. hierzu F. G. Carnell, Political Implications of Federalism in New States, in: U. K. Hicks u. a., Federalism and Economic Growth in Underdeveloped Countries, London 1961, S. $16 \mathrm{ff}$., S. $21 \mathrm{ff}$.

Ferner die oben bereits erwähnte Arbeit von Watts sowie C. Leys und P. Robson, Federation in East Africa, Nairobi und London 1965. Eine Sammlung der Texte von föderalistischen Verfassungen hat die

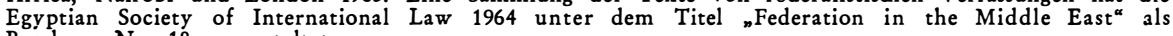
Brochure $\mathrm{Nr} .18$ veranstaltet.

68 So muß z. B. die Entscheidung für Wirtschaftsplanung, weil sie nur eine gesamtstaatliche Planung sein kann, auf den Bundesstaat im Sinne einer Straffung zugunsten des Gesamtstaates zurückwirken; vgl. Watts, a. a. O., S. 48.

og Es handelt sich um denselben Vorzug, der die Kommune vor der Korporation auszeichnet.
} 
rechtliche Problem des Minderheitenschutzes ${ }^{70}$ und die bekannten Methoden $\mathrm{zu}$ dessen Bewältigung. In diesem Zusammenhang verdient gesteigerte Aufmerksamkeit die Frage, wem man diesen Schutz aufträgt, ob insbesondere dieser Auftrag an die Gerichtsbarkeit, etwa eine besondere Verfassungsgerichtsbarkeit erteilt wird.

\section{Entpersönlichung und Entprivatisierung der Herrschaft}

Dasjenige Element moderner Staatlichkeit, das am schwierigsten zu verbreiten und $\mathrm{zu}$ rezipieren sein dürfte, ist das Selbstverständnis der Allgemeinheit als Institution, deren Gehalt die Idee des Gemeinwesens ist, womit persönliche und private Herrschaft ganz und gar unvereinbar ist ${ }^{71}$. Hiervon ist bereits früher etwas angeklungen, als der Gedanke eines dominium über das Staatsgebiet verworfen wurde. Da sich die Möglichkeit persönlicher oder privater Herrschaft hierin selbstverständlich nicht erschöpft, gilt es jetzt das Auge für alle diejenigen Variationen zu schärfen, in welchen persönliche oder private Herrschaft innerhalb eines Gemeinwesens auftreten kann, wobei Vollständigkeit zu erreichen angesichts der Unerschöpflichkeit der menschlichen Erfindungsgabe gerade in dieser Hinsicht von vornherein als unmöglich angesehen werden muß.

Die Eliminierung des persönlichen Momentes aus der staatlichen ${ }^{72}$ Herrschaft stößt sogleich auf die Schwierigkeit, daß vielfach die neuen Staaten ihre Existenz starken Führern verdanken und daß vor allem staatliche Herrschaft ohne eine Komponente von Führerschaft schon im allgemeinen, vor allem aber für die neuen Staaten unvollständig und unwirksam wäre ${ }^{73}$. Unter diesen Umständen wäre eine gänzliche Entpersonalisierung der Herrschaft, selbst wenn sie sich verwirklichen ließ $\mathrm{e}^{74}$ nicht nur impraktikabel, sondern auch durchaus systemwidrig. Das Problem erweist sich damit als ein solches der Grenzziehung zwischen legitimer Personalisierung der Innehabung und Ausübung der höchsten Staatsämter und illegitimem „Personenkult“. Mehr als daß es eine solche Grenze gibt und daß sie dort verläuft, wo die Institution endet und die Willkür beginnt, wird sich hierzu schwerlich sagen lassen.

Aus dem Bereich der Politik läßt sich erst recht das personale Moment nicht vollständig ausklammern. Die spezifischen Bildungen dieser Sphäre, insbesondere die politischen Parteien, bedürfen geradezu per definitionem eines Führers, der als Person sichtbar und greifbar macht, was das Programm meint, und der die Bürger qua Person anspricht und gewinnt ${ }^{75}$. Auch hier ist alles eine Frage der Grenzziehung. Eine „Partei“, die bei Lichte besehen nichts anderes ist als ein Führer und seine persönliche Gefolgschaft liegt zweifellos jenseits der Grenze der Legitimität. Das entsprechende gilt für den Sinn, den man mit Politik verbindet. Als extremes historisches Beispiel dafür, was nicht mehr als Politik verstanden werden

70 In Malaysia hat sich die Notwendigkeit eines Schutzes der (malaischen) Mehrheit vor den Minderheiten, insbesondere vor den mobileren und agileren Chinesen ergeben; vgl. T. E. Smith, The Background to Malaysia, London 1963.

11 Wesentlichkeit und Unerläßlichkeit der „dépersonalisation du pouvoir ${ }^{\star}$ in einem als Institution verstandenen Modernen Staat ist das Leitmotiv des von Georges Burdeau verfaßten großen Traité de Science Politique.

72 Wie man es mit nichtstaatlicher Herrschaft in dieser Hinsicht zu halten hat, soll offenbleiben.

73 Man erinnere sich daran, daß man vom amerikanischen Präsidenten selbstverständlich und einhellig leadership " fordert.

76 Bekanntlich entscheiden sich die Wähler nicht für ein Programm, sondern für das nächste Regierungshaupt; vgl. Sir Ivor Jennings, The British Constitution, 5. Aufl., Cambridge 1966, S. 162.

75 Hierin liegt der Grund, warum die afrikanische Politik "more personal" ist; so David E. Apter, The Political Kingdom in Uganda, Princeton 1961, S. 302, Anm. 1; vgl. hierzu für Afrika A. J. Richards, in: The King's Men, Oxford 1964, S. VII. 
darf, bietet der Kardinal de Retz ${ }^{76}$. Hier ist Politik lediglich Befriedigung einer Lust an der Intrige und deren Perfektion - ein technisch vollendetes Spiel ohne jeden sachlichen Sinn.

Persönliche Herrschaft oder persönliche Politik sind nicht ohne weiteres gleichzusetzen mit privater Herrschaft und privater Politik. Privat meint in diesem Zusammenhang zweierlei: Einmal die Möglichkeit daß die Träger staatlicher Ämter oder politischer Funktionen sich als Privatleute mißverstehen, und zweitens die Nutzung solcher Ämter und Funktionen zum privaten Vorteil. Ein solches Fehlverständnis kann hervortreten bereits bei der Erlangung und Behauptung öffentlicher Ämter und Funktionen. In der zweiten Hinsicht bietet ein bemerkenswertes Beispiel der sogenannte "continuismo“ der iberoamerikanischen Politik, d.h. die Kunst, ein (selbstverständlich unter persönlichen und privaten Vorzeichen innegehabtes) Amt auch nach dem Ablauf der Amtszeit zu behalten ${ }^{77}$. Solche Symptome deuten nicht nur auf ein Mißverständnis, sondern geradezu auf das Fehlen von Staatlichkeit hin. Was sich hier Staat und Politik nennt, ist in Wirklichkeit ein Kampf um Macht und Pfründen, der sich zwischen Personen oder Personengruppen an der Oberfläche der Gesellschaft abspielt und das Volk gleichgültig läßt. Es bedarf kaum der Hervorhebung, daß solche Zustände von Tag zu Tag unhaltbarer werden ${ }^{78}$. In einem Zeitalter, das durch die Revolution der steigenden Erwartungen gekennzeichnet ist, ist die Masse nicht mehr zur Resignation bereit, sie erwartet vielmehr soziale Leistungen und einen entsprechend leistungsfähigen und leistenden Staat. Da oligarchische Cliquen hierfür nicht einmal ein Organ haben, dürfte ihre Verdrängung durch echte Staatlichkeit nur noch eine Frage der Zeit sein.

Im Rahmen des Kapitels „Persönliche Herrschaft zu privaten Zwecken“ werden neben der Figur des "Berufspolitikers“ vor allem zwei Phänomene, nämlich $\mathrm{K}$ orruption ${ }^{79}$ und Patronage, ins Auge zu fassen sein. Was die erste angeht, so hat man sie nicht zuletzt als eine vormoderne Methode anzusehen, Amtstätigkeit in Gang zu setzen und zu dem gewünschten Ergebnis zu bringen ${ }^{80}$. Für die Allgemeine Staatslehre kommt es hier in erster Linie darauf an, ob die neuen Staaten die Idee des Amtes übernehmen und welches Motiv sie wählen, um der Amtstätigkeit Antrieb und Richtigkeit zu verleihen. Es handelt sich mit anderen Worten um die Frage, ob ein Staatsdienst eingerichtet und wie er verstanden wird - eine Frage, deren Beantwortung durch die neuen Staaten für Europa besonders wichtig und interessant ist, weil hier die Idee des Amtes und eines eigenartigen Staatsdienstes sich in einer schweren Krise befindet. Das Stichwort „Patronage“ lenkt die Aufmerksamkeit sowohl auf die Auswahl der Kandidaten für die Parlamentssitze ${ }^{81}$ wie für die Besetzung der staatlichen Ämter. Die Ausmerzung von Patronage ist eines der konkreten Ziele, das den Kampf um die Allgemeinheit des Staatsbürgertums gegen "Tribalismus“, überhaupt gegen die überkommenen Sozialstrukturen rechtfertigt ${ }^{82}$.

\footnotetext{
76 Memoires, Paris 1961.

77 Vgl. hierzu vor allem F. D. Parker, The Central American Republics, Oxford 1964, S. 83, 188. Dieser Autor berichtet auch von einem mittelamerikanischen Staatspräsidenten, der zu Ende seiner Amtszeit Eigentümer von 10 Prozent des Volksvermögens war: a. a. O., S. 245.

78 Das Auftreten der Úberzeugung, daß Armut nicht Schicksal, sondern von Menschen verursacht und daher auch durch den Menschen überwindbar ist, ist die Folge der Begegnung mit dem Westen, nicht zuletzt mit dem westlichen Film; vgl. von Vorys, a. a. O., S. 84, und (für den Film) S. 56.

$79 \mathrm{Vgl}$. R. Wraith und E. Simpkins, Corruption in Developing Countries, London 1964.

so Die Überwindung der Korruption mag daher zunächst zu einer Verschlechterung der Arbeitsweise führen. Vgl. hierzu die in den Denkwürdigkeiten L. von Gerlachs, II (1892), S. 19, berichrete Reaktion des Fürsien Dolgorucki auf die Nachricht, Kaiser Alexander würde allen Mißbräuchen in Rußland ein Ende machen: "Dieu l'en préserve, le désordre et la confusion est l'élément dans lequel nous existons."

81 Zum Beweis dafür, wie es in dieser Hinsicht noch im Europa des 19. Jahrhunderts bestellt war, sei verwiesen auf Cornelius O'Leary, The Elimination of Corrupt Practices in British Elections 1869-1911, verwiesen auf Cornelius O'Leary, The Elimination of Corrupt Practices in British Elections 1869-1911,
Oxford 1962.

82 Zur Vervollständigung des Bildes sei darauf hingewiesen, daß nicht nur die Herrscher, sondern auch die
} 
Hiermit sind einige der wichtigsten Punkte bezeichnet, die eine Verfassungsvergleichung aus der Sicht der Allgemeinen Staatslehre aufs Korn zu nehmen hat. Was die Entpersonalisierung und Entprivatisierung von Herrschaft und Politik angeht, so findet sich zwar in den neuen Gemeinwesen Asiens und Afrikas wohl nur in seltensten Fällen Herrschaft zum persönlichen Nutzen des Herrschers und seiner Umgebung etwa im Stile der albanischen Herrscher Ägyptens ${ }^{83}$. Wohl aber ist die Herrschaft hier vielfach eine Herrschaft von Personen, deren Charisma sich im Befreiungskampf gebildet und diesen naturgemäß überdauert hat. Beispiele hierfür bieten sich in allen neuen Erdteilen so zahlreich und so ausgesprochen an - man denke nur an die individualisierenden Bezeichnungen wie Adipadi oder Osagyefo ${ }^{84}$ - daß auf eine Aufzählung verzichtet werden kann ${ }^{85}$.

Eine Verfassung braucht einen solchen Sachverhalt nicht einmal in Andeutungen widerzuspiegeln, zumal sie als ein Gefüge abstrakt-allgemeiner Normen schon von Hause aus wenig geeignet ist, ihm förmlich Ausdruck zu verleihen. Bemerkenswert ist es daher, daß in der Verfassung von Ghana 1960 eine namentlich bezeichnete Person zum ersten Präsidenten bestimmt wird ${ }^{86}$, und daß durch Artikel 55 dieser Persönlichkeit besondere Vollmachten erteilt werden. Die Verjagung dieses Mannes zeigt allerdings, daß man auch an Ort und Stelle die Grenzen sieht, die der persönlichen und privaten Pervertierung von Herrschaft und Politik gezogen sind, und daß man eine Überschreitung dieser Grenze nicht duldet.

Die hier versuchte Bezeichnung derjenigen Themen deren Beobachtung die Staatstheorie fordert, ist weit davon entfernt, vollständig zu sein. Im Zusammenhang mit der Allgemeinheit des Staatsbürgertums z. B. stellen sich zwei Probleme, deren theoretischer und praktischer Stand im Westen schwere Sorgen bereitet. Es handelt sich einmal um die äußere und innere Teilnahme („participation“) auch des letzten Bürgers am Gemeinwesen - die für die Demokratie eine Existenzvoraussetzung ist. Die zweite Crux bildet der Gehorsam gegenüber dem Gesetz, vor allem gegenüber dem (in der Demokratie) selbstgegebenen Gesetz. Im Westen hat sich die seltsame These verbreitet, daß die Freiheit nicht etwa das um der Menschenwürde willen an jedermann verliehene Privileg darstelle, einen des Zusammenlebens wegen unvermeidlichen Gehorsam aus freiem Entschluß leisten und den Inhalt dieser Leistung in der Wahl und auf anderen Wegen frei bestimmen zu können -, daß vielmehr Gehorsam gegenüber dem Gesetz und persönliche Freiheit in Widerspruch stünden und daß daher Ungehorsam das erste Gebot der Freiheit sei. Womit die neuen Staaten in dieser Hinsicht zu rechnen haben, ist nicht nur der begreifliche Mangel an moderner Bürgergesinnung; es finden sich hier vielmehr auch scheinbar recht zähe Residuen aus der Zeit des Kampfes gegen die Kolonialherren. So hält es z. B. K. M. Pannikar ${ }^{87}$ für erforderlich, seine Mitbürger darauf aufmerksam zu machen, daß grundsätzlicher Widerstand gegen die Staatsgewalt nicht mehr als Tugend angesehen werden kann, seitdem er sich nicht mehr gegen die Engländer, sondern gegen die eigene Nation richtet. Nicht mehr von nachmodernem Widerstand, sondern von vormoderner Selbsthilfe, Fehde und Blutrache muß man sogar sprechen, wenn man von

Beherrschten den Staat als Pfründe mißbrauchen können; vgl. hierzu C. A. Almond und S. Verba, The Civic Culture, Princeton 1963, S. 83: Eine kinderreiche mexikanische Mutter erinnert sich des Staates nur dann, wenn es ihr darauf ankommt, einen ihrer Sprößlinge durch Unterbringung im Staatsdienst zu versorgen. 83 zu versorgen.

83 Vgl. hierzu Naday Safran, Egypt in Search of Political Community, Cambridge, Mass., 1961, S. 108.

85 Eine besonders plastische Formulierung für diese Herrschaft der Person findet sich bei Khalid Ben Sayeed, Pakistan, The formative phase, Karachi 1960, S. 408: "To the people of Pakistan, Jinnah was Pakistan."

86 Vgl. Art. 10: "Kwame Nkrumah is hereby appointed first President of Ghana."

87 The State and the Citizen, 2. Aufl., London 1960, S. 2; Shils, a. a. O., S. 34, stellt hierzu fest, der Befreiungskampf habe geschaffen eine Tradition von "demonstrative ${ }^{\alpha}$ und "remonstrative politics“. 
K. von Vorys erfährt ${ }^{88}$, daß gemäß der in Pakistan herrschenden Auffassung wohl der Privatmann Gewalt anwenden darf, nicht jedoch der Staat - geschweige denn daß von einer Monopolisierung der Gewalt in den Händen des Staates die Rede sein könnte.

\section{Themen aus der Verfassungslehre}

Die Hoffnungen, die die Verfassungslehre ${ }^{89}$ in die Beschäftigung mit Verfassungsrecht und Verfassungswirklichkeit in Übersee setzt, reichen über die Gewinnung von Varianten bekannter Typen weit hinaus: Hier geht es um nicht mehr und nicht weniger als eine kritische Sicht des klassischen westlichen Modells selbst, und zwar nicht nur um der neuen, sondern vor allem auch um der alten Staaten willen. Trotz aller schon zurückgelegten Fortschritte bleiben die westlichen Nationen sich in Zivilisation und Technik unablässig weiter modernisierende Nationen und dies ungeachtet des seltsamen Umstandes, daß ihre Verfassungen niemals eigentliche Modernisierungsverfassungen gewesen sind und es heute erst recht nicht sind. Diese Diskrepanz hat man wohl in erster Linie darauf zurückzuführen, daß der sogenannte Verfassungsstaat in Kontinentaleuropa erst in einem Augenblick ins Leben getreten ist, als die Aufgabe der Modernisierung - vor allem wenn man sie als OKkonomisierung versteht ${ }^{90}$ - im Begriff war, vom Staat auf die Gesellschaft überzugehen -, wenn man nicht sogar diesen Verfassungsstaat als die Konstitutionalisierung eines ausschließlichen Rechts der Gesellschaft auf Vollführung der Modernisierung zu verstehen hat. Insbesondere wird man die in allen diesen Verfassungen verbürgten Grundrechte durchaus auch als Ausschaltung des Staates aus der Modernisierung und Vorbehalt dieses Prozesses zugunsten der Gesellschaft verstehen können.

Ist dies richtig, dann wäre die kritische Frage, zu der die Verfassungsvergleichung anregt, dahin zu formulieren, ob der Westen die Ausschaltung des Staates aus der (weiteren) Modernisierung aufrechterhalten kann. Offensichtlich ist diese Frage in der Praxis schon längst mit „Nein“ beantwortet worden - wie insbesondere die aktive Rolle zeigt, die der Staat gegenüber und in der Wirtschaft spielt. Hier ist die Ideologie hinter der Wirklichkeit zurückgeblieben, während sie in den neuen Staaten den Ereignissen vorauseilt. Wie es sich aber immer in allen diesen Hinsichten verhalten mag: Dort wo die Modernisierung erst in Angriff genommen wird, kann es ebenso wie seinerzeit in Europa nur der Staat sein, der sie als entscheidende Stelle betreibt. Hieraus ergibt sich für die Betrachtung der politischen Verfassungen der neuen Staaten, daß sie vor allem darauf durchzusehen sind, ob sie Modernisierung als Staatsaufgabe und als Verfassungsziel proklamieren und ob sich insbesondere eine solche Verfassung als Aufruf zur Mitwirkung an dieser erhabenen Aufgabe erweist. Hat man mit einer solchen Eigenart der neuen Verfassungen zu rechnen, dann muß man sich als erstes und wichtigstes klarmachen, daß das Wesen einer Verfassung keineswegs in der Grenzziehung zwischen Privatautonomie und Staatsherrschaft besteht - daß ein solches Verfassungsverständnis vielmehr ein durchaus bedingtes ist und daß der Sinn einer Verfassung sehr wohl auch in der Instituierung und Ordnung von staatlicher Aktivität und von Zusammenwirken liegen kann. In

88 A. a. O., S. $46 \mathrm{ff}$

80 Im Sinne des angelsächsischen Sprachgebrauches ist hierunter sowohl "Government" wie "Political Science" verstanden.

эo Vgl. hierzu Léon Epsztein, L’Économie et la Morale aux Débuts du Capitalisme Industriel en France et en Grande-Bretagne, Paris 1966, S. $19 \mathrm{ff}$. 
einem solchen Falle, in dem die Verfassung den Staat von der eigenen Leistung nicht fern-, sondern zu einer solchen anzuhalten und zu befähigen hat, wird man eine konkrete Verfassung vor allem daraufhin zu mustern haben, welche Vorkehrungen sie trifft, um die Handlungs- und Leistungsfähigkeit des Staates zu steigern. Einen ersten Hinweis darauf, daß man es mit einer Modernisierungsverfassung im Sinne einer Verfassung, die zu Modernisierung aufruft und sie zugleich organisiert und aktiviert, zu tun hat, gibt die Feststellung, daß man eine „flexible“ zum Unterschied von einer "rigiden" Verfassung"1 vor sich hat: Da eine Modernisierung immer wieder vor unvorausgesehenen Situationen steht, ist ihr nur eine Verfassung angemessen, die elastisch genug ist, um alsbald und adäquat reagieren $\mathrm{zu}$ können. Entscheidend für die Charakterisierung einer Verfassung ist das Verhältnis, in das sie die drei Gewalten zueinander stellt. Jedenfalls nicht für Modernisierung hat man sich entschieden, wenn man die parlamentarische Gesetzgebung an die erste Stelle setzt, und geradezu als gegen die Modernisierung gerichtet muß eine Entscheidung für den Justizstaat gewertet werden. Positiv gewendet sind somit Modernisierungsverfassungen dadurch gekennzeichnet, daß sie Regierung und Verwaltung eine starke Stellung einräumen ${ }^{92}$. Da eine solche Stellung der Exekutive im Westen vielfach als anstößig empfunden wird, obwohl jedenfalls in einer freiheitlichen Demokratie hierin nichts Anstößiges liegen kann, tut man gut, sich daran zu erinnern, daß Verfassungsvergleichung nicht im Messen konkreter Verfassungen an einem abstrakt-universalen Verfassungsideal und noch weniger in der Erteilung von Zensuren wegen Abweichungen besteht, daß sie vielmehr zunächst einmal Verständnis für die konkreten Verfassungen erwecken soll, indem sie sie auf die Notwendigkeiten zurückführt, die die Lagen vorzeichnen, in denen sich der betreffende Staat befindet. Nur ein solches Verständnis vermag das zu vermitteln, worauf allein es der Verfassungsvergleichung ankommen muß: Nicht Selbstbestätigung oder gar Selbsterhebung, sondern Bereicherung der Erkenntnis.

1. Das $S t a$ a t $s$ oberha u $t$ ist unter zwei Aspekten zu beobachten, die kurz als „Repräsentation“ und als „Funktion“ angesprochen sein sollen. „Repräsentation" bezieht sich auf den Staat, und zwar den Staat als Ganzheit und als Einheit. $\mathrm{Da}$ wo der Zusammenhang des Staatsverbandes ohnehin weitgehend gesichert ist, können sich die Gründe, die für eine schwache Stellung des Staatsoberhauptes geltend gemacht werden, sehr viel besser durchsetzen als dort, wo dies wie in den neuen Staaten nicht der Fall ist: Ein Präsident, der die „tragende Stütze des (afrikanischen) Staatsgebäudes" ist ${ }^{93}$, kann nur ein Präsident sein, dessen verfassungsrechtliche ${ }^{94}$ Stellung eine starke ist: Die Persönlichkeit allein vermag Repräsentation und Integration des Staates nicht zu bewirken.

Was die Funktion angeht, so liegt die Vermutung nahe, daß die gesteigerte Bedeutung der Exekutive dem Staatsoberhaupt zugutekommen werde. Dieser Schluß erweist sich jedoch nicht als zwingend. Gerade wenn man die Gewichtigkeit der nicht eigentlich exekutiven Aufgaben des Oberhauptes eines solchen neuen Staates ermißt, kann es sich empfehlen, den Schwerpunkt der Exekutive in die Regierung zu legen. Auch in diesem Falle stellt sich allerdings die Frage, wo man die „Reservegewalt" zu suchen hat, die dann hervortreten soll, wenn die normalen Zuständig-

\footnotetext{
o1 Diese Unterscheidung geht zurück auf J. Bryce, Studies in History and Jurisprudence I, Oxford 1901, S. $196 \mathrm{ff}$.

92 Vgl. hierzu für den Westen P. M. Gaudemet, Le Pouvoir Exécutif dans les Pays Occidentaux, Paris 1966. 93 So Eugen Fehr, a. a. O., S. 141.

os Es ist hierbei also nicht an Anomalien von der Art gedacht, daß eine Person zugleich Staatsoberhaupt, Regierungschef und Führer der einzigen Partei ist, wie dies für Kwame Nkruma zugetroffen hat; vgl. Leslie Rubin, The Constitution of Ghana, in: Jahrbuch des Offentlichen Rechts der Gegenwart - N. F. Bd. XIV, Tübingen 1965, S. $585 \mathrm{ff}$.
} 
keiten und Befugnisse auf außergewöhnliche Umstände und Ereignisse stoßen. Wenn es richtig ist, daß der indische Präsident nach dem Vorbild der englischen Königin geschaffen ist ${ }^{95}$ und daß damit auch die ungeschriebenen Befugnisse des Vorbildes rezipiert sind ${ }^{96}$, dann scheint das Ergebnis schlüssig, daß ein solcher Präsident latent über außerordentliche Vollmachten verfügt ${ }^{97}$.

Um noch beispielhaft eine Einzelheit zu bringen: Nicht zuletzt an das Staatsoberhaupt wird man die Frage zu richten haben, wem die Hut der Verfassung anvertraut ist, ob man also etwa in ihm den Hüter der Verfassung zu erblicken hat ${ }^{98}$.

Die Regierung im institutionellen Sinne ist ein Gegenstand, dem man im Westen, abgesehen etwa von Rudolf Smend ${ }^{99}$ und Georges Burdeau ${ }^{100}$, nicht diejenige Beachtung schenkt, die heute insbesondere die Steigerung und Veränderung der Anforderungen an den Staat vor allem in wirtschaftspolitischer Hinsicht an sich verlangten. Ganz allgemein gesprochen läßt sich hierzu ohne weiteres das folgende sagen: Wenn das Bedürfnis nach stärkerer Aktivität der Exekutive nicht dem Staatsoberhaupt zugutekommt, dann kann es nur die Regierung sein, die sich seiner anzunehmen hat. Dies braucht keineswegs als etwas neues, das klassische Modell des Verfassungs- und Rechtsstaates alterierendes Moment angesehen zu werden. In England jedenfalls war man immer undoktrinär genug, in einer starken Regierung die erste Voraussetzung für eine wirksame Staatlichkeit zu sehen. Es handelt sich heute nicht mehr nur darum, daß das "Politische“101 einen Mittelpunkt haben muß, der ihm Berücksichtigung gegen den Zug zur Entpolitisierung und Einfluß auf die an sich unpolitischen Staatstätigkeiten sichert. Es ist gerade in den neuen Staaten die Eigenart der Ziele, die das Beharren in einem reinen Normativismus und der Reduktion der Regierung auf bloße "Exekutive“ nicht mehr gestattet. B. de Jouvenel102 hat in diesem Zusammenhang von einer Ersetzung der "Nomokratie“ durch die "Telokratie“ gesprochen.

Von hier aus fällt bereits ein erstes Licht auf das Thema „P a r l a me n t“: Wenn die Modernisierung als Ziel der neuen Staaten eine handlungs- und leistungsfähige Regierung fordert, dann muß hier die seltsamerweise so gut wie gar nicht behandelte Frage, welches die genuinen Aufgaben eines Parlamentes sind ${ }^{103}$, mit der alten englischen Formel beantwortet werden, daß das Parlament nicht sei ein "governing“, sondern ein „government-making body“. Darüber hinaus wird man in solcher Sicht das Parlament als ein Element der Regierung (im funktionellen Sinne) anzusehen haben insofern als es sich um die demokratische Erarbeitung der Strategie der großen Politik handelt. Gerade hierauf wird die Vergleichung zu achten haben, wenn sie einen Beitrag zur Revision einer sehr zeitbedingten Auffassung von der Beschränkung der Parlamente auf Normsetzung leisten will. Nicht übersehen kann sie aber auch die Versuche, die Bedeutung der Parlamente herabzudrücken, die unter den Parolen einer "controlled“, "guided" oder "tutelary" democray, insbesondere eines „rationalisierten“ oder "regulierten“ Parlamentarismus, in den neuen Staaten

\footnotetext{
$95 \mathrm{Vgl}$. J. L. Kapur, The Status and the Powers of the President of India, in: Jahrbuch des Offentlichen Rechts - N. F. - Bd. XIV, Tübingen 1965, S. 57 ff., S. 58.

96 Kapur, a. a. O., S. 67, 68 f.

97 Kapur, a. a. O.

08 Bereicherung der verfassungstheoretischen Einsicht verspricht insbesondere auch die vergleichende Beschäftigung mit dem Problem, welche Art der Bestellung des Präsidenten man in den republikanischen Staaten gewählt hat und welche Uberlegungen für eine solche Wahl maßgebend gewesen sind.

99 Die politische Gewalt im Verfassungsstaat und das Problem der Staatsform, in: Festgabe der Berliner juristischen Fakultät für Wilhelm Kahl, Tübingen 1923.

100 Traité de Science Politique.

$101 \mathrm{Vgl}$. hierzu jetzt vor allem Julien Freund, L'Essence du Politique, Paris 1965.

102 Zitiert nach P. Avril, Le Régime Politique de la Ve République, 2. Aufl., Paris 1965, S. 18.

103 Man pflegt durchweg ohne jeglichen Zweifel dem Parlament Gesetzgebung als vornehmste, wenn nicht im Grunde sogar als einzige Aufgabe zuzusprechen, was historisch sicher falsch, aktuell aber ungenau ist.
} 
umgehen. In dieser Hinsicht wird speziell darauf zu achten sein, ob man dem Parlament die Gesetzgebungsbefugnis schlechthin oder nach dem Muster der französischen Verfassung von 1958 nur für bestimmte spezielle Materien zugesprochen hat - mit der Wirkung, daß im übrigen die Normsetzung Sache der Regierung oder der Verwaltung ist ${ }^{104}$.

Es dürfte heute in der ganzen Welt kaum noch einen Staat geben, der nicht Wert darauf legte, sich als $\mathrm{De}$ mok $\mathrm{r}$ a $\mathrm{i}$ e zu bezeichnen und als solche international anerkannt zu sein. Da es jedoch von vornherein ausgeschlossen ist, daß - abgesehen vom Wort - unter diesen Umständen Demokratie gleich Demokratie sein könnte, muß für das Staatsformenproblem hieraus der Schluß gezogen werden, daß man nicht mehr mit Aristoteles zwischen Monarchie, Aristokratie und Demokratie zu unterscheiden, sondern innerhalb der Demokratie typische Gestaltungen aufzusuchen hat -, was im übrigen der Terminus "Volksdemokratie“ nahelegt. Bedeutet daher Demokratie zunächst einmal Allgemeinheit des Staatsbürgertums im quantitativen Sinne, dann wird man für eine dergestalt gestellte Frage nach der Staatsform zuerst darauf abzuheben haben, ob diese Allgemeinheit wirklich bis zum letzten Bewohner und zur letzten Bewohnerin des betreffenden Staates ausgedehnt ist und auf welche Art und Weise sie verwirklicht wird. Der erste Umstand macht sich kenntlich durch das Fehlen von Privilegien und die ausnahmslose Gleichheit der Rechte und Pflichten, insbesondere der Grundrechte und Grundpflichten. In der zweiten Hinsicht ist wichtig die Art der Aktualisierung dieses allgemeinen Status, also ob sie durch Plebiszite, durch Wahlen oder auf eine sonstige Art und Weise erfolgt. Letztlich kommt es darauf an, ob man von einer wahrhaften, wenn auch dialektischen Identität von Volk und Staat reden darf oder ob autonome Positionen der Autorität prätendiert werden, die nicht vom Volke ausgehen und daher der Einwirkung durch das Volk nicht unterliegen, wie das in Frankreich die Fünfte Republik voraussetzt und hierauf einen eigenständigen Staat gründet, als dessen Repräsentant General de Gaulle auftritt. Eine solche Konstruktion wirkt vor allem vor dem Hintergrund der Demokratie jedenfalls zunächst einigermaßen rätselhaft und bedarf daher besonders sorgfältiger Durchdringung.

Es liegt auf der Hand, daß gerade in diesem Zusammenhang die Berücksichtigung der realen Machtfaktoren nicht unterlassen werden darf. Von Auftreten des miles perpetuus an haben hierher an erster Stelle die W e h r m a ch te $\mathrm{n}$ gerechnet und ihnen hat daher traditionell die Abneigung des Liberalismus und der liberalen Demokratie - vor allem der Angelsachsen - gegolten ${ }^{105}$. Hierzu ist als erstes festzustellen, daß es in allen neuen Staaten of t beachtliche, so weit wie möglich modern ausgerüstete Wehrmachten gibt, obwohl die Notwendigkeiten der Verteidigung nicht immer ohne weiteres erkennen lassen, daß hiermit einem echten Bedürfnis der Landesverteidigung entsprochen wird. Es muß unter diesen Umständen zum mindesten vorsorglich die Frage nach einer innenpolitischen Bedeutung dieser nationalen Wehrmachten aufgeworfen werden ${ }^{106}$. Man muß damit rechnen, daß die Antworten auf diese Frage sehr verschieden ausfallen können, und zwar sogar für ein und dasselbe Land in den verschiedenen Epochen seiner Geschichte ${ }^{107}$. Es ist

104 Zur Frage, ob etwa der Parlamentarismus überhaupt reformbedürftig sein könnte, vgl. J. Chapsal, La Vie Politique en France depuis 1940, Paris 1966, S. 540.

$105 \mathrm{Vgl}$. hierzu etwa E. V. Rostow, The Sovereign Prerogative: The Supreme Court and the Quest for Law, 1962, S. XXII, und E.-O. Czempiel, Das amerikanische Sicherheitssystem 1945-1949, Berlin 1966, S. 355.

106 Vgl. hierzu im allgemeinen M. Janowitz, The Military in the Political Development of New Nations, Chicago 1964.

107 Dies ergibt sich aus John J. Johnson, The Military and Society in Latin America, Stanford 1964. Eine Ubersicht über die Staatsstreiche der Militärs in Lateinamerika gibt G. Connell-Smith, The Inter-American System, Oxford 1966, S. 183. 
daher wegen der Vermeidung vorschneller Verallgemeinerungen zu begrüßen, daß eine beachtliche Anzahl von Spezialuntersuchungen zu diesem Thema vorliegt ${ }^{108}$.

Zwei Eventualitäten sind in diesem Rahmen auf jeden Fall ins Auge zu fassen. Es handelt sich einmal um eine spezielle Affinität des heutigen Soldaten zur Modernität. Ob ein Zivilist sich auf einem Kamel oder in einem Kraftwagen fortbewegt, mag noch einigermaßen in der Freiheit seiner Entschließung liegen. Zwischen Pfeil und Bogen einerseits und Maschinenwaffen andererseits hat der Soldat keinerlei Wahl -, er ist vital zur extremsten und letzten Modernität der Bewaffnung und Ausrüstung gezwungen. Wie seinerzeit in Kontinentaleuropa vom miles perpetuus die Modernisierung und insbesondere ihr Produkt und Vehikel, der Moderne Staat ausgegangen ist, so verlangt auch in den neuen Staaten die Modernität der Armee die Modernisierung des Gesamtlebens in allen seinen Bereichen. Der Soldat stellt zugleich den Prototyp des modernen Menschen dar, der den Geist der Modernisierung in alle anderen Schichten trägt ${ }^{109}$.

Als zweite Möglichkeit muß man damit rechnen, daß eine über die militärischen Angelegenheiten hinausgehende Stellung oder Rolle der Wehrmacht sich aus dem Sachverhalt „Korruption" ergeben kann: Es hat den Anschein, als ob zuweilen der Soldat der am wenigsten korrupte, ja sogar vielleicht der einzige nicht-korrupte Bürger seines Vaterlandes wäre ${ }^{110}$. Man darf daher die Erklärung nicht von vornherein ausschließen, daß eine Machtübernahme durch die Armee darauf zurückzuführen ist, daß die Korruption der zivilen Machthaber einen Grad erreicht hatte, der ein weiteres Zusehen nicht mehr duldete.

Abgesehen von dem Ertrag, den die Beschäftigung mit diesem Gegenstand für ein besseres Verständnis der neuen Staaten und ihren Eigenarten verheißt, verspricht sie auch wertvolle Ausbeute für die Deutung des Verhältnisses von ziviler und militärischer Gewalt überhaupt ${ }^{111}$. Die größten Rätsel gibt wahrscheinlich auch in dieser Hinsicht China dem Betrachter auf ${ }^{112}$.

2. Ein Streifzug durch die Themen, die die Verfassungswirklichkeit der Vergleichung stellt, stößt auch auf unserem Feld zuerst auf die politis chen $\mathrm{Par}$ t e i e ${ }^{113}$. Das ist keine Selbstverständlichkeit: Denn das Bemühen eines Volkes, sich für seine Herrschaft auf demokratische Weise aktionsfähig zu machen, mußte als Ergebnis keineswegs die politische Partei zeitigen. Auf der anderen Seite muß bedacht werden, daß die Feststellung der Existenz von politischen Parteien nicht ohne weiteres dahin gedeutet werden darf, daß sie dieselbe Rolle spielen wie in Europa. Man hat sich vielmehr daran zu erinnnern, daß diese Parteien vielfach eher bestanden und gewirkt haben als der Staat bestanden hat, in dem wir sie heute vorfinden. Ihr Sieg mag dann bedeuten, daß „the party takes over the State" 114 oder daß gar eine solche Partei sich als Staat konstituiert ${ }^{115}$. Selbst wenn man aber eine

${ }^{108}$ Vgl. insbesondere die beiden Sammelwerke John J. Johnson, The Role of the Military in Underdeveloped Coutries, Princeton 1962, und Léo Hamon, Le Role extra-militaire de l'Armée dans le tiers monde, Paris 1966.

109 Vgl. zu alledem Shils, a. a. O., S. 24, Anm. 2; und Apter, Modernization, S. 135, Anm. 18.

110 Vgl. P. C. Lloyd (ed.), The New Elites of Tropical Africa, Oxford 1966, S. 9: Nur das Militär hat saubere Hände; ferner von Vorys, a. a. O., S. 146, 147.

111 Vgl. Malcolm Kerr, The Arab Cold War 1958-1964, Oxford 1965, S. 4: “ . . the chronic involvement of the Syrian army in politics..."

Im Irak hat die Armee mehrfach gefordert, zur Regierung zugelassen zu werden, weil sie allein über die Fähigkeit und den Willen zu Reformen verfüge; vgl. Majid Khadduri, Independent Iraq - A Study in Iraqui Politics from 1932 to 1958, 2. Aufl., Oxford und London 1960, S. 74, 106.

112 Vgl. F. Schurmann, a. a. O., S. 187: Die Armee in China "has become a vital part of civil organization...". Hierzu insbesondere John Gittings, The Role of the Chinese Army, Oxford 1967.

113 Vgl. hierzu vor allem Ruth Schachter Morgenthau, Political Parties in French-Speaking West Africa, Oxford 1964; ferner etwa Welch, a. a. O., S. 11 ff.; R. L. Sklar, Nigerian Political Parties, Princeton 1963. Die Geschichte einer Partei gibt z. B. John A. Martz, Acción Democrática - Evolution of a Modern Political Party in Venezuela, Princeton 1966

11 D. E. Apter, Uganda, S. 302.

115 Apter, a. a. O., S. 306; ferner Nehru, bei Hanson, a. a. O., S. 33: “. . Now that the Congress is, to some extent, identifying itself with the State..." 
solche Partei als Selbstveranstaltung eines souveränen Volkes zur Ausübung seiner demokratischen Herrschaft begreift, darf man nicht ohne weiteres voraussetzen, die Dynamik eines solchen Parteiwesens müsse dieselbe sein, wie der europäische Verfassungstypus sie im Sinne hat. Insbesondere muß man sich hier wie überall der Neigung enthalten, das Phänomen durch die Brille von „competitiveness“ zu sehen ${ }^{116}$ und über eine etwa festgestellte Wettbewerbslosigkeit ein Unwerturteil zu fällen. Da die einzige Partei nach Sy ${ }^{117}$ den Schlüssel zum Verständnis der afrikanischen Zustände liefert ${ }^{118}$, würde man sich hierdurch nicht nur der Möglichkeit eines Erfassens dieser Zustände berauben, sondern sich auch die Beobachtung der Wirkungen unmöglich machen, die das Einparteiensystem auf das rezipierte europäische Verfassungsmodell ausübt ${ }^{119}$.

Da Einzigkeit des Daseins und der Gewalt ein wesentliches Merkmal des Staates ist, deutet ein Einparteiensystem darauf hin ${ }^{120}$, daß eine solche Partei weniger Partei als Staat sein könnte. In der Tat wird von China berichtet, hier verhalte sich Partei zu Staat wie Regierung zu Verwaltung ${ }^{121}$, ja die staatliche Rechtspflege sei schlechthin durch die Partei verdrängt worden ${ }^{122}$. Eine Staatsaufgabe wird gewiß einer solchen Partei nicht vorenthalten sein, nämlich den Willen zur Modernisierung $\mathrm{zu}$ wecken sowie einen entsprechenden Gesamtwillen zu bilden und durchzusetzen ${ }^{123}$. Hierfür mag nicht zuletzt auch sprechen, daß eine politische Partei als ein ursprünglich gesellschaftliches Gebilde eher als der Staat die Mittel der „Uberredung" einzusetzen vermag, um die Massen für eine Politik der Modernisierung zu gewinnen ${ }^{124} 125$.

Die Beschäftigung mit den Parteien, lenkt unwillkürlich den Blick auf die „P o li tike r". Das klassische westliche Verfassungsmodell überantwortet die Politik bekanntlich nicht Amtsträgern oder Funktionären einer staatsartigen Partei. Sie setzt hierfür in ihre Konstruktion ein vielmehr eine besondere Art von Menschen, eben den "Politiker“, d. h. eine von einer Einordnung in eine Institution oder gar Hierarchie freie Persönlichkeit, die sich entschlossen hat, einen guten Teil ihrer Zeit und ihrer Kraft, ja vielleicht sogar ihr Leben dem Dienst an der Politik zu widmen, wobei das Motiv eines solchen Entschlusses durchaus auch ein eigennütziges wie z. B. Befriedigung von Ehrgeiz und Machttrieb sein darf. Fehlt es an Honoratioren, die von ihrem Vermögen leben, dann bildet sich der Typ der Berufspolitiker ${ }^{126}$ heraus, dem die Sorge für das tägliche Brot auf eine Art und Weise abgenommen werden muß, die jede Korruption ausschließt. Da dieses Problem selbst in den Mutterländern dieses Verfassungsmodells nicht völlig befriedigend bewältigt ist, stellt sich für die neuen Staaten um so dringlicher die Frage, ob und wie ihnen die Lösung dieses Problems gelungen ist - zumal man hier gewiß noch der natür-

116 So aber J. S. Coleman bei G. A. Almond - J. S. Coleman (ed.), The Politics of the Developing Areas, Princeton 1960, S. 533: ncompetitiveness is an essential aspect of political modernity “. Diese Behauptung kann nur schlicht als falsch bezeichnet. werden, so sehr sie auch dem Westen selbst als Ideal vorkommen mag, das er gegen den Osten zu verteidigen hat.

117 A. 2. O., S. 12.

118 Vgl. hierzu G. M. Carter (ed.), African One-Party States, Ithaca, N. Y., 1962.

$110 \mathrm{Vgl}$. die oben Anm. 30 erwähnte Arbeit von Kliesch.

120 Nach sowjetrussischer Auffassung bedarf es mehrerer Parteien nur dort, wo es mehrere widerstreitende Klasseninteressen gibt. Ist daher wie in einem kommunistischen Staat nur ein einziges wirtschaftliches Interesse vorhanden, dann bedarf es auch nur einer Partei; vgl. J. N. Hazard, The Soviet System of Government, Rev. Ed., Chicago 1960, S. 72.

121 F. Schurmann, a. a. O., S. 110.

122 F. Schurmann, a. a. O., S. 188.

123 Hierzu Apter, Modernization, S. 360; ferner Young, a. a. O., S. 329: "The mass single-party, not the state incarnated the sovereign 'general will' of the people."

126 Vgl. hierzu außer Apter, a. a. O., S. 363, vor allem C. G. Ströhm, Zwischen Mao und Chruschtschow Wandlungen des Kommunismus in Südostasien, Stuttgart 1964, S. 111.

$125 \mathrm{Zu}$ alledem ist schließlich noch $\mathrm{zu}$ verweisen auf A. R. Zolberg, One-party Government in the Ivory Coast, Princeton 1961.

18 Vgl. hierzu Max Weber, Politik als Beruf, 2. Aufl., München und Leipzig 1926. 
lichen Auffassung im gleichen Maße begegnet, wie sie sich in Europa noch bis zum 19., ja 20. Jahrhundert, findet, wozu anders eine Position da sein könne als zum eigenen oder der Familie Nutzen ${ }^{127}$.

Die Frage nach den Politikern weist wiederum zurück auf das Problem einer $f \ddot{u} \mathrm{~h}$ -

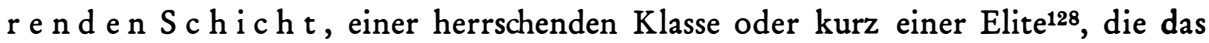
Reservoir bilden könnte, aus dem sich die Politiker rekrutieren, ohne daß selbstverständlich hiermit die Funktion einer Elite erschöpft wäre. Hierzu läßt sich eine allgemeingültige Aussage machen: Überall steht es fest, daß die traditionellen Führungsschichten als Elite und insbesondere als Reservoir von Politikern nicht in Betracht kommen, da die Modernisierung, die die Politiker betreiben sollen, sich ja gerade gegen die „Häuptlinge“, den „Feudalismus“ oder wie immer man die Gegenposition disqualifizieren mag, zu richten hat. Diese Konstellation macht neue Eliten notwendig, zugleich aber ihre Bildung schwierig und daher ihre Existenz zweifelhaft - ganz abgesehen davon, daß die Frage, ob es eine führende Schicht gibt und welche Gruppe man als solche anzuerkennen hat, heute auch für den "Westen“ nicht eben einfach zu beantworten ist. Stichworte, die unter solchen Umständen der Untersuchung eine erste Orientierung vermitteln könnten, sind etwa Bürokratie, Technokratie und vor allem Intelligentsia. In diesem Zusammenhang wird man sich auch der These Lavroff $\mathbf{s}^{129} \mathrm{zu}$ erinnern haben, daß das politische Leben in einigen neuen Staaten nichts anderes ist als der Kampf mehrerer neuer Eliten um die Macht.

Gerade wenn man bedenkt, daß Rezeption niemals sklavische Nachahmung sein kann, wird man darauf achten müssen, ob die politische Selbstformierung und Selbstaktivierung des demokratischen Volkes sich weniger der Gestalt der Partei bedient, als daß es andere Bildungen vorzieht ${ }^{130}$. Es muß aber auch mit der Möglichkeit gerechnet werden, daß diese Rolle von einer Organisation gespielt wird, die dem Namen und der Art nach auch im Westen bekannt ist, deren spezifische Funktion hier aber eine andere ist. Gedacht ist hierbei vor allem an die G e w e r k $\mathrm{s} \mathrm{ch}$ a f t e n ${ }^{131}$. Während des Kampfes gegen die Kolonialherren haben sie zuweilen die politische Rolle gespielt, die an sich die Parteien hätten spielen müssen ${ }^{132}$. Der Europäer wird hinsichtlich dieser Gebilde Beobachtungen vor allem unter jenem Gesichtspunkt anzustellen haben, den man in Europa "Soziale Frage" nennt. Den Kern dieser Frage bildet das Problem, eine neue, zahlreiche Art von Bürgern, nämlich den Industriearbeiter, in Staat und Gesellschaft zu integrieren, also ihnen das Bewußtsein der "Zugehörigkeit" zu beidem und als Voraussetzung hierfür „Soziale Sicherheit" zu vermitteln. Die neuen Staaten haben die Chance, aus den europäischen Erfahrungen, ja Versäumnissen vor allem die Lehre zu ziehen, daß sie am besten tun, diese Frage gar nicht erst entstehen zu lassen. Das heißt: Wenn man die Menschen im Zuge der industriellen Revolution aus ihren traditionellen Strukturen herausreißt, dann darf man diese „befreiten“ Individuen nicht sich selbst überlassen - es ist vielmehr ein Gebot der Selbsterhaltung dieser neuen Staaten und Gesellschaften, zugleich mit der "Befreiung" neue soziale Strukturen die alte Aufgabe der staatlichen und gesellschaftlichen Integration übernehmen zu lassen.

${ }^{127}$ Hieran erinnert noch die deutsche Wendung, daß "ein Amt seinen Mann zu nähren" habe.

128 Vgl. P. C. Lloyd (ed.), The New Elites of Tropical Africa, London 1966, mit allerdings etwas ungleichartigen und ungleichwertigen Beiträgen; ferner Th. Kerstiens, The new Elite in Asia and India, New York 1966.

128 Bei Sy, a. a. O., S. 5.

130 Einige Beispiele hierfür bei Young, a. a. O., S. $379 \mathrm{f}$

131 Vgl. hierzu Myron Wiener, The Politics of Scarcity - Public Pressure and Political Response in India, Chicago 1962; S. C. Sufrin, Unions in Emerging Societies - Frustration and Politics, Syracuse, N. Y., 1964.

132 Hierzu etwa W. J. Foltz, From Frend West Africa to the Mali Federation, New Haven 1965, S. 21; und Stahn, a. a. O., S. 24. 
Es liegt auf der Hand, welch wichtige Aufgabe in diesem Rahmen den Gewerkschaften zufällt, sofern nämlich diese Neuintegration in einem freien Staate wesentlich und vor allem Selbstintegration sein muß, die naturgemäß nur eine durch besondere Gruppen vermittelte, also kollektive, sein kann. Hier liegt die erste und schwerste Verantwortung der Gewerkschaften sofern sie sich richtig als „Ordnungsmächte" verstehen. Dieser sozialen Funktion vermögen Gewerkschaften aber nur zu genügen, wenn sie und ihre Mitglieder sich nicht lediglich als passive Nutznießer der Modernisierung und ihrer Früchte verhalten, sondern bereit sind, eine Mitverantwortlichkeit für beides zu übernehmen. Im Westen hat die Ausstattung der Sozialpartner mit Tarifautonomie vor allem den Sinn, ihnen die Möglichkeit der Bewährung einer solchen Mitverantwortung in ihrem eigensten Bereich, im Bereich der "Arbeitsbedingungen" einzuräumen. Daß diese Mitverantwortung weiter geht, $\mathrm{da} ß$ sie insbesondere auch die soziale Integration des Industriearbeiters einschließt, sollte in den neuen Staaten noch weniger übersehen werden als in den alten, weil man sich dort sehr viel weniger auf anderweitige, die Allgemeinheit zusammenhaltende Bande verlassen kann.

\section{Verwaltung, Verwaltungsrecht und Dienstrecht}

Eine Modernisierung durch das Vehikel des Modernen Staates verlangt vor allem eine moderne Verwaltung. Das gilt insbesondere auch für die Modernisierung der Wirtschaft: Heute ist es ein Gemeinplatz, daß Wirtschaftshilfe sinnlos ist, wenn sie nicht von der Schaffung einer entsprechenden "Infrastruktur" begleitet wird ${ }^{\mathbf{1 3 3}}$. Man pflegt daher den Vorsprung derjenigen Staaten hervorzuheben, die im Augenblick ihrer Entstehung einen fähigen Staatsdienst von Landsleuten übernehmen und sogleich einsetzen konnten ${ }^{134}$.

Ein Staat, der "Modernisierung" auf seine Fahnen geschrieben hat, kann daher nach Regierungsstaat - nur Verwaltungsstaat sein. In dieser Feststellung sind zwei Verneinungen enthalten: Was einmal das Verhältnis zum Recht angeht, so kann dieser Staat seine Aufgabe nicht darin sehen, Rechtssätze und Berechtigungen lediglich deswegen zu bewahren, weil sie überkommen oder wohlerworben sind - ein solcher Staat kann nicht "Rechtsbewahrungsstaat" sein; er muß in beiden Hinsichten rechtsschöpferisch im Sinne einer Verbindung von Fruchtbarkeit und Gerechtigkeit tätig werden können. Eben diese Verpflichtung gegenüber dem lebendigen Recht ist es auch, die es ausschließt, daß ein sich modernisierender Staat Justizstaat sein könnte, also ein Staat, der das letzte Wort in allen, auch in den politischen Fragen der Rechtspflege anvertraut. Eine solche Verneinung des Rechtsbewahrungsund des Justizstaates wird vielen Europäern anstößig sein, denen die Erhaltung der überkommenen Verfassungs- und Rechtszustände wie die Bewahrung der erworbenen Rechte die Staatsaufgabe schlechthin zu sein scheint und die deswegen geneigt sind, über sich modernisierende Staaten harte Unwerturteile zu fällen. Um so mehr ist es gerade an dieser Stelle erforderlich, sich der Zeit-, um nicht zu sagen der Klassenbedingtheit ${ }^{135}$ dieser Auffassung bewußt und sie daher nicht etwa zum Maßstab zu machen, an dem gemessen Moderne Staaten nur als Nichtrechtsstaaten oder gar als Unrechtsstaaten erscheinen könnten.

\footnotetext{
133 Hierzu Hanson, a. a. O., S. 268: "Unfortunately, underdeveloped societies tend, almost by definition, to have underdeveloped administration."

134 Vgl. hierzu A. L. Adu, The Civil Service in New African States, New York 1965.

195 L. Duguit hat darauf hingewiesen, daß die Verfechter dieser Auffassung die feudalen Besitzstände keineswegs geachtet und geschont haben.
} 
Wenn Modernisierung vor allem OKonomisierung bedeutet, dann müssen Wirtschaftspolitik und eine sie verwirklichende Wirtschaftsverwaltung eine hervorragende Stelle in der wissenschaftlichen Aufmerksamkeit beanspruchen. Einmal handelt es sich hier um die Frage, an welchem Vorbild sich ein neuer Staat in dieser Hinsicht orientiert. So sachlich die Antwort auf diese Frage im konkreten Falle bestimmt sein mag, so ist eine solche Entscheidung doch immer zugleich auch eine weltpolitische insofern als die Auseinandersetzung zwischen Ost und West vor allem eine Auseinandersetzung um Richtigkeit und Sittlichkeit der beiderseitigen, angeblich diametral entgegengesetzten Wirtschaftsmodelle ist. Auch an dieser Stelle ist daher die Unbefangenheit der wissenschaftlichen Würdigung gefährdet: Um so mehr muß sie darauf bedacht sein, die Wirtschaftsverfassung und Wirtschaftspolitik betreffenden Entscheidungen eines neuen Staates weniger ideologisch als aus den Forderungen der Lage zu erklären.

Dies letzte gilt vor allem für das Thema „Planung“. Für viele Ideologen gleichermaßen im Osten wie im Westen ist dies das Schibboleth, an dem sich die Geister scheiden. Selbstverständlich kann hiervon im Ernst keine Rede sein. So sehr die Ideologie an solchen Entscheidungen beteiligt sein mag - den Ausschlag gibt doch immer die Notwendigkeit der Lage und die Qualität des Modells an sich, also seine Effizienz, die im übrigen nicht unbedingt und in erster Linie eine ökonomische zu sein braucht: Man sollte sich daher den Weg zu wahrhafter Erkenntnis durch die Annahme nicht verlegen, daß Wirtschaften notwendig nur im Dienste von wirtschaftlichen Zielen stehen könne.

Eine durch solche Zielsetzungen und die ihr gemäße Dynamik geprägte Verwaltung ist es, die das Bild des öffentlichen Dienstes vorzeichnet ${ }^{136}$. Es kann kein Zweifel daran bestehen, daß nur der Leistungsgrundsatz unter diesen Umständen das ihn bestimmende Prinzip sein kann - wobei sich gerade hier die Frage stellt, ob nicht für die Wirtschaftspolitik und die Wirtschaftsverwaltung von einer besonderen Art der Leistungsfähigkeit die Rede sein müßte, die sich Initiative, Phantasie und Elastizität in einem höheren Maße verpflichtet weiß, als dies im allgemeinen der Fall ist. Dieses Leistungsprinzip ist der sehr nüchterne Grund, der Aufbau und Förderung eines Bildungswesens zum umungänglichen Gebot macht. Schließlich kommt es auch bezüglich der Feststellung der Leistungsfähigkeit zu denjenigen Einrichtungen, die Europa hierfür entwickelt hat - Examen und Versachlichung der Auswahl durch besondere Kommissionen ${ }^{137}$.

\section{Aufruf zu Teilnahme und Mitarbeit}

Diese Zeitschrift entspringt noch weniger fertig den Köpfen ihrer Väter als irgendeine andere: Sie muß im wahrsten Sinne des Wortes erst werden, was sie sein soll. Man könnte sich sogar fragen, ob selbst unter den günstigsten Bedingungen und trotz der bereitesten Mitarbeit ihr letztes und eigentliches Ziel zu erreichen ist -, eine weltweite Vergleichung von Verfassung und Recht. Dies gilt um so mehr, als die Ergebnisse dieser Forschung und Vergleichung wiederum verglichen werden sollen mit den westlichen Gestaltungen in ihrer Typik. Denn Arbeiten, die einen bestimmten, aus Verfassung und Recht entnommenen Gegenstand durch die westlichen Verfassungs- und Rechtsordnungen hindurch verfolgen, sind selten ${ }^{138}$, und

\footnotetext{
${ }^{136}$ Hierzu K. Younger, Public Service in the New States, Oxford 1960.

137 Vgl. die Civil Service Commission der englischen Tradition.

138 Vgl. etwa Robert A. Dahl, Political Opposition in Western Democracies, Yale UP 1966, und F. Castberg, Freedom of Speach in the West, Oslo-London 1960.
} 
selbst diese Ausnahmen sind mehr Juxtapositionen als Darstellungen der typischen Regularitäten und der atypischen Irregularitäten.

Solche Schwierigkeiten rechtfertigen es jedoch nicht, von einem Vorhaben abzusehen, wie es hier begonnen wird. Man redet heute so viel und so gern von „weltweiten Verflechtungen“ des Verkehrs, der Wirtschaft und der Politik. Ist dies zweifellos richtig, dann verlangt es nicht nur die Vollständigkeit, daß auch die Wissenschaften, und zwar insbesondere auch Staats- und Rechtswissenschaft, in eine weltweite Kommunikation treten. Wenn man ebenso mit vollem Recht fordert, daß die Völker sich gründlicher kennenlernen müßten, um sich besser zu verstehen, dann ist die Vermittlung solcher wechselseitiger Bekanntschaft gewiß nicht nur Sache von Reiseunternehmen, sondern in erster Linie Aufgabe von Publizistik und vor allem Wissenschaft. Nicht ohne Sehnsucht gedenkt man allerdings heute der Zeit, da die Gelehrten lateinisch schrieben und sich deswegen wenigstens sprachlich verständigen konnten. Doch auch die sprachlichen Schwierigkeiten von heute sollten kein unüberwindliches Hindernis der Kenntnisnahme von einander und der Verständigung untereinander bilden.

Das Unternehmen, Verfassung und Recht in Übersee zur Kenntnis zu bringen, den Austausch solcher Erkenntnisse zu vermitteln und sie vergleichend für Staats- und Rechtswissenschaften auszuwerten, muß daher gewagt werden. Mehr als andere bedarf ein so weitgespannter und anspruchsvoller Versuch der Hilfen aller Art, angefangen mit Anregungen in jeglicher Hinsicht. Dieses Programm schließt daher folgerichtig mit einem Aufruf an jedermann zu Teilnahme und Mitarbeit. Die Herausgeber werden sich bemühen, den Helfern jede Erleichterung zu verschaffen ${ }^{139}$. Der Widerhall, den dieser Aufruf findet, wird nicht zuletzt darüber entscheiden, ob und wie dieses Unternehmen sich verwirklicht.

139 Hierher gehört auch der Entschluß, dieser Zeitschrift "Beihefte ${ }^{\alpha}$ zuzuordnen, um auch größeren Arbeiten die Publizität zu sichern. Das erste dieser Hefte ist bereits erschienen: Enno Kliesch, Der Einfluß des französischen Verfassungsdenkens auf afrikanische Verfassungen (vgl. oben Anm. 30). 